\title{
Adaptação do HCM2000 para análise da capacidade e do nível de serviço em rodovias de pista simples no Brasil
}

\author{
José Reynaldo Setti ${ }^{1}$, José Elievam Bessa Júnior ${ }^{2}$, \\ Cíntia Yumiko Egami ${ }^{3}$ e Márcia Lika Mon-Ma ${ }^{4}$
}

\begin{abstract}
Resumo: A meta deste trabalho foi descrever, de maneira prática, como aplicar uma adaptação do método do HCM2000 para análise da capacidade e do nível de serviço em rodovias de pista simples brasileiras. A adaptação proposta resulta de duas pesquisas que, basicamente, replicaram os procedimentos usados no desenvolvimento do método original do HCM, substituindo os dados obtidos em rodovias dos EUA e Canadá por dados coletados em dez trechos de rodovias brasileiras. Dessa forma, são apresentadas relações entre a taxa de fluxo, a velocidade média de viagem e a porcentagem de tempo seguindo em pelotões, além de tabelas com fatores de ajuste para condições não básicas. Podem ser avaliados segmentos longos e homogêneos, numa análise em sentido duplo ou em sentido único, além de rampas específicas. Os resultados obtidos indicam que as estimativas dos níveis de serviço usando-se a adaptação do método do HCM2000 tendem a refletir melhor as reais condições operacionais das vias brasileiras de pista simples.
\end{abstract}

Palavras-chave: nível de serviço, capacidade, rodovia de pista simples, simulação de tráfego.

\begin{abstract}
The goal of this study was to describe, in a practical way, how to apply an adaptation of HCM2000 method for capacity and level of service analysis on two-lane rural highways. The proposed adaptation is the result of two research projects that basically replicated the procedures used for developing the original HCM method, while replacing the data collected on highways in the U.S. and Canada for data from ten sections of Brazilian highways. Thus, relationships between flow rate, average travel speed and percent timespent-following are presented, as well as tables containing adjustment factors for situations differing from base conditions. It is possible to estimate the level of service of long homogeneous segments, considering one-way or two-way flows, as well as specific upgrades. The results indicate that the level of service estimates obtained with the use of the HCM2000 adaptation tend to reflect better the actual highway conditions.

Keywords: level of service, capacity, two-lane rural highways, traffic simulation.
\end{abstract}

\section{INTRODUÇÃO}

O Highway Capacity Manual (HCM), cuja quinta versão foi lançada em dezembro de 2010 (TRB, 2010), é a principal referência usada no mundo para analisar a capacidade e o nível de serviço em rodovias. No Brasil, avaliações desse tipo são frequentemente realizadas com base no HCM2000 (TRB, 2000), a quarta versão do manual. A principal alteração do HCM2010, em relação ao HCM2000, foi o abandono da análise para fluxos nos dois sentidos. Para determinar o nível de serviço nesses casos, deve-se realizar uma combinação das medidas de desempenho obtidas a partir dos modelos voltados para um único sentido.

O HCM tem sido recomendado por órgãos brasileiros para analisar as condições operacionais em rodovias e para determinar a necessidade de obras de melhoramento (ARTESP, 2002; DNIT, 2006; TCU, 2008). No entanto, o HCM é, geralmente, aplicado no Brasil sem nenhuma adaptação às condições locais. Uma vez que a maior parte da pesquisa básica foi realizada com dados de rodovias ameri-

${ }^{1}$ José Reynaldo Setti, Departamento de Engenharia de Transportes, Escola de Engenharia de São Carlos, Universidade de São Paulo, São Paulo, SP, Brasil. (e-mail: jrasetti@usp.br).

2 José Elievam Bessa Júnior, Departamento de Engenharia de Transportes, Escola de Engenharia de São Carlos, Universidade de São Paulo, São Paulo, SP, Brasil. (e-mail: jebessa@sc.usp.br).

${ }^{3}$ Cíntia Yumiko Egami, Departamento de Engenharia de Transportes, Escola de Engenharia de São Carlos, Universidade de São Paulo, São Paulo, SP, Brasil. (e-mail: cintiaye@gmail.com).

${ }^{4}$ Márcia Lika Mon-Ma, Agência Nacional de Transportes Terrestres, Brasília, DF, Brasil. (e-mail: marcia.mon-ma@antt.gov.br).

Manuscrito recebido em 18/4/2011 e aprovado para publicação em $30 / 5 / 2011$. Este artigo é parte de TRANSPORTES v.19, n.2, 2011. ISSN: 2237-1346 (online). canas e canadenses, o próprio manual adverte que sua aplicação em outras regiões requer alterações das equações e dos processos de modo que representem adequadamente o comportamento nesses lugares (TRB, 2000; TRB, 2010).

Essa necessidade provém de diversos fatores que são diferentes em relação às observadas na América do Norte. Dentre essas condições distintas, destaca-se a forma de condução dos motoristas (agressividade, obediência às regras de trânsito), as características dos veículos (tamanho, potência, massa) e a composição veicular (DNIT, 2006). Adaptações do HCM foram desenvolvidas em diversos países com o intuito de melhor refletir as particularidades locais, o que culminou, inclusive, em completas modificações do processo de estimativa, como na Alemanha (Brilon e Weiser, 2006) e na África do Sul (Van As, 2003).

Diversos estudos foram elaborados visando produzir conhecimentos que auxiliem na adaptação do HCM para o Brasil. Para rodovias de pista simples, as primeiras tentativas de adaptação foram baseadas na premissa de que a estrutura do HCM, versão publicada em 2000, deveria ser modificada apenas no que fosse necessário (Egami, 2006; Mon-Ma, 2008). Esses trabalhos, desenvolvidos concomitantemente usando dados coletados em estradas paulistas, se complementam, formando uma adaptação quase completa do método proposto no Capítulo 20 do HCM2000. Estudos subsequentes mostraram que essa adaptação produz resultados mais confiáveis que a aplicação direta do manual (Utimura et al., 2007). Este artigo visa mostrar, de maneira prática, como aplicar a adaptação proposta para análise da capacidade e do nível de serviço em rodovias de pista simples no Brasil. 


\section{VISÃO GERAL DO MÉTODO DE ADAPTAÇÃO}

O método usado para adaptar o Capítulo 20 do HCM2000 consistiu, basicamente, em replicar os procedimentos documentados em Harwood et al. (1999), substituindo-se os dados de rodovias dos EUA e Canadá por dados coletados em rodovias no estado de São Paulo. Como os procedimentos envolvem o uso de simulação, um aspecto importante da adaptação foi a calibração de simuladores microscópicos de tráfego de rodovias de pista simples para o Brasil, realizado por meio de um Algoritmo Genético (AG), uma técnica de busca e otimização inspirada no mundo biológico e que se baseia na teoria de evolução de Darwin (Goldberg, 1989). O AG é um método iterativo orientado para a solução de problemas complexos, com muitas variáveis, como é o caso da calibração de simuladores de tráfego.

O algoritmo se inicia com a produção aleatória de um conjunto de soluções compostas pelos parâmetros do simulador. Durante cada iteração, as soluções são avaliadas e selecionadas segundo uma função objetivo (fitness) definida com base nas medidas de desempenho simuladas e observadas em campo. As medidas de desempenho usadas na aplicação do AG foram a velocidade média de percurso e a porcentagem de veículos em pelotões. Ao longo do processo, os indivíduos que fornecem as melhores soluções permanecem na população transmitindo as suas características, enquanto que os menos adaptados tendem a desaparecer. Maiores detalhes sobre a estratégia de calibração usada podem ser obtidos em outros trabalhos (Egami et al., 2006; Egami, 2006; Mon-Ma, 2008).

Foram coletados dados de tráfego em 10 trechos de rodovias no estado de São Paulo, com uma ampla gama de características geométricas e de tráfego (Egami et al., 2006; Mon-Ma, 2008). Cada trecho foi observado duas vezes, para compor dois conjuntos de observações, um para ser utilizado na calibração e outro na validação do simulador. Com os simuladores calibrados, foram realizadas simulações com combinações de taxas de fluxo, de velocidades de flu- xo livre, de relevo e de composição do tráfego, com o intuito de adaptar relações fundamentais (entre fluxo, velocidade e porcentagem de tempo seguindo em pelotões), tabelas com fatores de ajuste e outras equações, como a usada para estimar a velocidade de fluxo livre. Os fatores de ajuste foram encontrados por meio das diferenças entre as medidas de desempenho para condições básicas e não básicas.

$\mathrm{O}$ item a seguir mostra como usar, de maneira prática, a adaptação realizada. As equações e as tabelas apresentadas seguem, na maior parte dos casos, a mesma estrutura sugerida pelo HCM2000. Os valores das fórmulas e das tabelas, no entanto, foram modificados, a fim de refletir a realidade das estradas observadas.

\section{ADAPTAÇÃO DO HCM2000 PARA RODOVIAS DE PISTA SIMPLES}

As medidas de desempenho usadas para determinar o nível de serviço em rodovias de classe I, ou seja, aquelas em que os motoristas esperam viajar com altas velocidades, são a velocidade média de viagem (ATS) e a porcentagem de tempo seguindo em pelotões (PTSF). A medida utilizada em rodovias de classe II, que são aquelas em que os motoristas não necessariamente esperam viajar a altas velocidades, é somente a PTSF. Os critérios para definição do nível de serviço em rodovias de pista simples estão apresentados na Tabela 1. No caso da classe I, a PTSF e a ATS devem ser atendidas simultaneamente, como mostra a Figura 1.

Esses critérios devem ser usados nas análises para um ou dois sentidos, para rampas específicas e com outras características, desde que fatores de ajustes sejam considerados. $\mathrm{O}$ nível de serviço " $F$ " é aplicado quando o fluxo de tráfego excede a capacidade, ou seja, $1700 \mathrm{cpe} / \mathrm{h}(\mathrm{cpe}=$ carros de passeio equivalentes) para um sentido do tráfego ou 3200 cpe/h quando a análise é em sentido duplo. A Figura 2 resume o procedimento para estimar o nível de serviço adotado pelo HCM2000. As seções a seguir mostram como a a-

Tabela 1. Critérios para determinação do nível de serviço em rodovia de pista simples de classe I e II (TRB, 2000)

\begin{tabular}{|c|c|c|c|c|}
\hline \multicolumn{3}{|c|}{ (a) Rodovias de classe I } & \multicolumn{2}{|c|}{ (b) Rodovias de classe II } \\
\hline$N S$ & PTSF (\%) & $\operatorname{ATS}(\mathrm{km} / \mathrm{h})$ & $N S$ & PTSF (\%) \\
\hline $\mathrm{A}$ & $\leq 35$ & $>90$ & $\mathrm{~A}$ & $\leq 40$ \\
\hline B & $>35-50$ & $>80-90$ & B & $>40-55$ \\
\hline $\mathrm{C}$ & $>50-65$ & $>70-80$ & $\mathrm{C}$ & $>55-70$ \\
\hline $\mathrm{D}$ & $>65-80$ & $>60-70$ & $\mathrm{D}$ & $>70-85$ \\
\hline E & $>80$ & $\leq 60$ & $\mathrm{E}$ & $>85$ \\
\hline
\end{tabular}

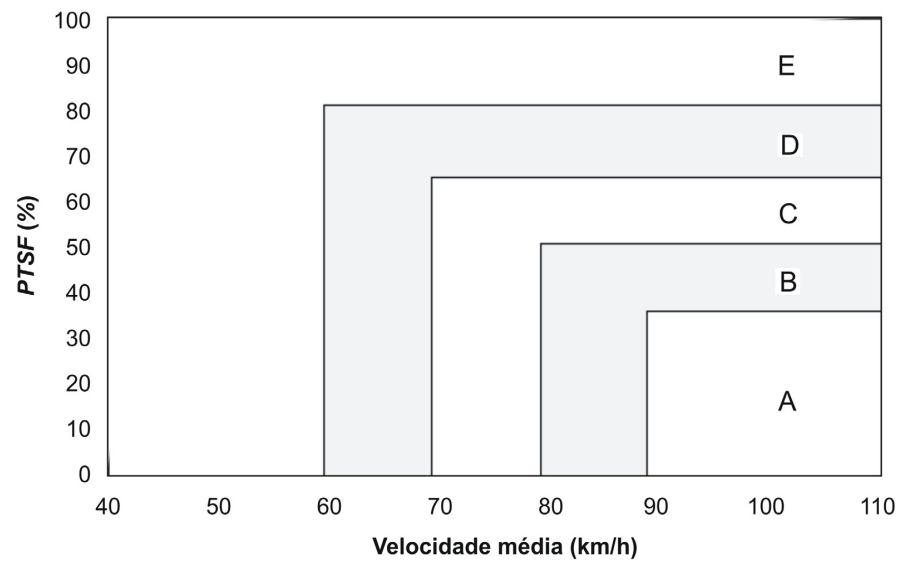

Figura 1. Critério para determinação do nível de serviço em rodovias de pista simples de classe I (TRB, 2000) 


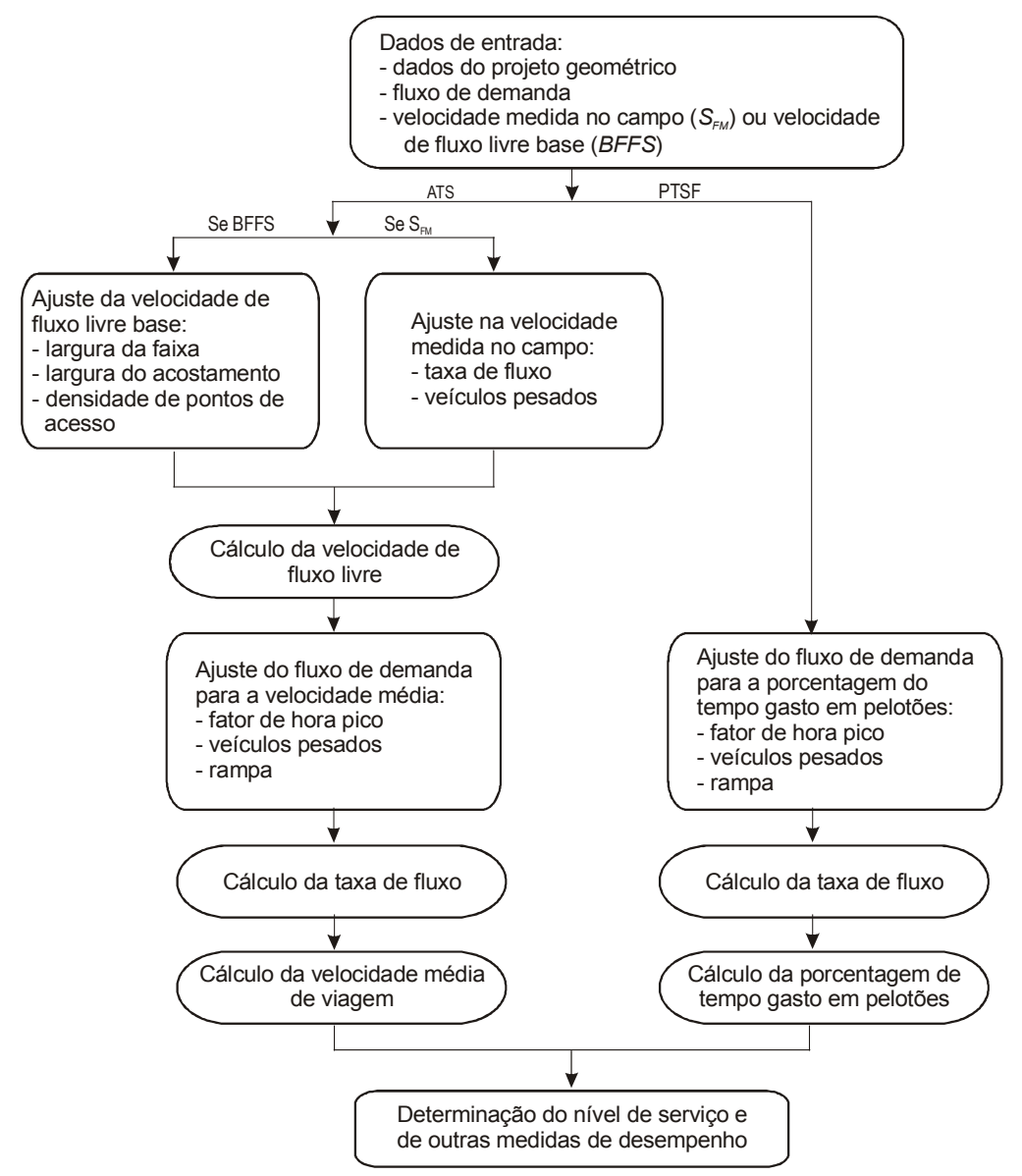

Figura 2. Método para estimativa do nível de serviço para rodovias de pista simples do HCM2000 (Egami, 2006)

nálise deve ser feita.

Método para estimativa do nível de serviço para rodovias de pista simples do HCM2000 (Egami, 2006)

\subsection{Segmentos em um ou dois sentidos}

A metodologia para avaliação de segmentos homogêneos, em sentido único ou em sentido duplo, pode ser usada para trechos onde o relevo é plano ou ondulado. As etapas para determinar o nível de serviço nesses casos são descritas nas subseções a seguir.

\subsubsection{Velocidade de fluxo-livre}

Considera-se a velocidade de fluxo livre a velocidade média de uma corrente de tráfego com taxa de fluxo nos dois sentidos de até $200 \mathrm{cpe} / \mathrm{h}$. Para fluxos maiores, existem duas maneiras de determinar a velocidade de fluxo livre, ou por meio da observação de correntes de tráfego em campo, ou realizando uma estimativa. A coleta de dados em campo deve ser realizada em um local que seja representativo do trecho avaliado. Nesse caso, deve-se usar:

$$
F F S=S_{F M}+0,0137 \cdot \frac{V_{f}}{f_{H V}}
$$

em que,

$F F S: \quad$ velocidade de fluxo livre estimada $[\mathrm{km} / \mathrm{h}]$;

$S_{F M}$ : velocidade média do tráfego medido em campo $[\mathrm{km} / \mathrm{h}]$;

$V_{f}$ : fluxo de tráfego observado no período em que os dados foram coletados [veic/h]; e
$f_{H V}$ : fator de ajuste para os veículos pesados.

No caso de impossibilidade para coletar dados em campo, uma estimativa para $F F S$ deve ser realizada por meio da expressão $F F S=B F F S-f_{L S}-f_{A}$, em que $B F F S$ é uma velocidade de fluxo livre base, entre 70 e $110 \mathrm{~km} / \mathrm{h}$ e escolhida com base na geometria da rodovia; e $f_{L S}$ e $f_{A}$ são, respectivamente, fatores de ajuste para a largura da faixa e do acostamento e para a densidade de pontos de acesso. Entretanto, no estado de São Paulo, a maior parte das rodovias de pista simples possui faixas de rolamento com largura de $3,6 \mathrm{~m}$ e acostamentos de $1,8 \mathrm{~m}$, o que, de acordo com o HCM2000, não alteram o desempenho do tráfego. Além disso, larguras diferentes para faixas de tráfego e acostamentos não podem ser simuladas, assim como a existência de pontos de acesso. Por essas razões, os fatores $f_{L S}$ e $f_{A}$ não foram tratados.

\subsubsection{Taxa de fluxo}

A taxa de fluxo de tráfego deve ser calculada em função da unidade cpe/h. Com isso, é determinado o impacto de outros tipos de veículos, como caminhões e ônibus, na corrente de tráfego. A Equação 2 mostra como desenvolver essa transformação:

$$
v_{p}=\frac{V}{P H F \cdot f_{G} \cdot f_{H V}}
$$

em que, 
$v_{p}$ : fluxo de carros de passeio equivalente no período de 15 minutos de pico dentro da hora de pico [cpe/h];

$V$ : fluxo horário na hora de pico [veic/h];

$P H F$ : fator de pico horário (Equação 3);

$f_{G}$ : fator de ajuste para rampas; e

$f_{H V}$ : fator de ajuste para os veículos pesados (Equação 4).

A taxa de fluxo $v_{p}$ pode ser relacionada com o sentido analisado, com o sentido oposto ao analisado ou para os dois sentidos. O fator de pico horário é dado por:

$$
P H F=\frac{v}{4 \cdot v_{15}}
$$

em que,

v: volume na hora de pico [veic/h]; e

$v_{15}$ : volume durante 15 minutos de pico [veic/15 min] dentro da hora de pico.

O fator de ajuste para o efeito de veículos pesados na qualidade de serviço é:

$$
f_{H V}=\frac{1}{1+P_{T} \cdot\left(E_{T}-1\right)+P_{R} \cdot\left(E_{R}-1\right)}
$$

em que,

$P_{T}$ : fração de caminhões e ônibus na corrente de tráfego, expresso em valores decimais;

$P_{R}$ : fração de veículos recreacionais na corrente de tráfego, em decimais;

$E_{T}$ : fator de equivalência veicular para caminhões e ônibus; e

$E_{R}$ : fator de equivalência veicular para veículos recreacionais.

No Brasil, é praticamente nula a presença de veículos recreacionais nas estradas. Esse fato levou à desconsideração dos impactos associados à presença desse tipo de veículo. Consequentemente, não há, na metodologia descrita neste trabalho, uma apresentação dos valores de $E_{R}$. Por outro lado, ônibus e caminhões são frequentemente observados nas rodovias brasileiras. O impacto desses veículos é representado pelo fator de equivalência veicular $E_{T}$, e deve ser determinado em função do fluxo de tráfego, do tipo de análise (um ou dois sentidos), do tipo de relevo e da medida de desempenho que se deseja estimar (ATS ou PTSF). O fator de ajuste $f_{G}$ determina o impacto das rampas na estimação das medidas de desempenho ATS e PTSF. Os valores de $E_{T}$ e $f_{G}$ para análise de segmentos em um ou em dois sentidos são apresentados nas Tabelas 2 e 3.

Até o momento, o método apresentado não permite determinar o valor do fluxo de tráfego dado em cpe/h. No entanto, essa informação é necessária para que os fatores de ajuste $E_{T}$ e $f_{G}$ possam ser determinados. Para solucionar esse problema, um método iterativo é proposto: primeiramente, calcula-se a relação entre o volume $V$, em veic/h, e o fator $P H F$. Com essa relação, determina-se os fatores $E_{T}$ e $f_{G}$ para, em seguida, estimar $v_{p}$ por meio da Equação 2. Se o valor de $v_{p}$ for menor do que o limite superior do intervalo de fluxos usado para determinar $E_{T}$ e $f_{G}$, deve-se usar o valor de $v_{p}$ calculado. Se for maior, o processo é reiniciado utilizando-se o intervalo de fluxo seguinte até que $v_{p}$ seja a-
Tabela 2. Fatores de ajuste $E_{T}$ e $f_{G}$ na determinação da ATS em segmentos em sentido único ou em sentido duplo

\begin{tabular}{llllll}
\hline $\begin{array}{l}\text { Taxa de fluxo } \\
\text { nos dois senti- }\end{array}$ & $\begin{array}{l}\text { Taxa de fluxo } \\
\text { em um sentido } \\
\text { dos }(\text { cpe/h) }\end{array}$ & \multicolumn{2}{l}{$\begin{array}{l}\text { Relevo } \\
\text { plane } / \boldsymbol{h})\end{array}$} & \multicolumn{3}{l}{$\begin{array}{l}\text { Relevo } \\
\text { ondulado }\end{array}$} \\
\cline { 3 - 6 } & $E_{T}$ & $f_{G}$ & $E_{T}$ & $f_{G}$ \\
\hline $0-600$ & $0-300$ & 5,9 & 1,0 & 4,3 & 0,72 \\
$>600-1200$ & $>300-600$ & 3,9 & 1,0 & 3,5 & 0,89 \\
$>1200$ & $>600$ & 2,4 & 1,0 & 2,4 & 0,93 \\
\hline
\end{tabular}

Tabela 3. Fatores de ajuste $E_{T}$ e $f_{G}$ na determinação da $P T S F$ em segmentos em sentido único ou em sentido duplo

\begin{tabular}{llllll}
\hline $\begin{array}{l}\text { Taxa de fluxo } \\
\text { nos dois senti- }\end{array}$ & $\begin{array}{l}\text { Taxa de fluxo } \\
\text { em um sentido }\end{array}$ & \multicolumn{2}{l}{$\begin{array}{l}\text { Relevo } \\
\text { plano }\end{array}$ (cpe/h) } & \multicolumn{2}{l}{$\begin{array}{l}\text { Relevo } \\
\text { ondulado }\end{array}$} \\
\cline { 3 - 6 } (cpe/h) & $E_{T}$ & $f_{G}$ & $E_{T}$ & $f_{G}$ \\
\hline $0-600$ & $0-300$ & 1,1 & 1,0 & 1,0 & 0,77 \\
$>600-1200$ & $>300-600$ & 1,1 & 1,0 & 1,1 & 0,87 \\
$>1200$ & $>600$ & 1,0 & 1,0 & 1,0 & 0,92 \\
\hline
\end{tabular}

ceito. Essas iterações também devem ser feitas na análise de rampas específicas.

\subsubsection{Relações fundamentais de tráfego}

As relações fundamentais de tráfego são aquelas entre a taxa de fluxo e as medidas de desempenho ATS e PTSF, usadas na determinação do nível de serviço em rodovias de pista simples. Essas relações foram obtidas para análises em dois sentidos e para um sentido, como mostram as Equações 5 a 8, sendo necessário saber o fator de ajuste para a porcentagem de zonas com ultrapassagens proibidas $\left(f_{n p}\right)$ e o fator combinado para a divisão entre os sentidos e para as zonas com ultrapassagens proibidas $\left(f_{d / n p}\right)$. Os valores dos coeficientes $a$ e $b$, necessários para obtenção de $P T S F_{d}$, são fornecidos na Tabela 4. As Tabelas 5 a 8 apresentam os valores de $f_{n p}$ e $f_{d / n p}$.

$$
\begin{gathered}
A T S=F F S-0,0098 \cdot v-f_{n p} \\
P T S F=100 \cdot\left(1-e^{-0,0011 \cdot v}\right)+f_{d / n p} \\
A T S_{d}=F F S_{d}-0,0137 \cdot v_{d}-0,0064 \cdot v_{o}-f_{n p} \\
P T S F_{d}=100 \cdot\left(1-e^{a \cdot v_{d}^{b}}\right)+f_{n p}
\end{gathered}
$$

em que,

$v$ : taxa de fluxo nos dois sentidos [cpe/h];

$v_{d}$ : taxa de fluxo no sentido analisado [cpe/h];

$v_{o}$ : taxa de fluxo no sentido oposto [cpe/h]; e

$a, b$ : coeficientes obtidos em função do fluxo oposto ao sentido analisado.

Tabela 4. Coeficientes a e $b$ para obtenção da $P T S F_{d}$

\begin{tabular}{lll}
\hline $\begin{array}{l}\text { Taxa de fluxo no } \\
\text { sentido oposto } \\
\text { (cpe/h) }\end{array}$ & \multicolumn{2}{l}{ Coeficientes } \\
\cline { 2 - 3 }$\geq 200$ & $a$ & $b$ \\
400 & $-0,0020$ & 0,9485 \\
600 & $-0,0064$ & 0,8088 \\
800 & $-0,0116$ & 0,7389 \\
1000 & $-0,0167$ & 0,6979 \\
1200 & $-0,0180$ & 0,6940 \\
1400 & $-0,0178$ & 0,7028 \\
$\leq 1600$ & $-0,0180$ & 0,7050 \\
\hline & $-0,0176$ & 0,7105 \\
\hline
\end{tabular}


Tabela 5. Fator de ajuste $f_{n p}$ na determinação da ATS em segmentos com sentido duplo

\begin{tabular}{lllllll}
\hline $\begin{array}{l}\text { Taxa de fluxo } \\
\text { nos dois senti- }\end{array}$ & \multicolumn{6}{c}{ Zonas de ultrapassagem proibida (\%) } \\
\cline { 2 - 7 } dos (cpe/h) & 0 & 20 & 40 & 60 & 80 & 100 \\
\hline 0 & 0,0 & 0,0 & 0,0 & 0,0 & 0,0 & 0,0 \\
200 & 0,0 & 0,2 & 0,4 & 0,6 & 0,9 & 1,5 \\
400 & 0,0 & 0,3 & 0,5 & 0,8 & 1,2 & 2,0 \\
600 & 0,0 & 0,4 & 0,6 & 1,0 & 1,4 & 1,9 \\
800 & 0,0 & 0,4 & 0,7 & 0,9 & 1,3 & 1,7 \\
1000 & 0,0 & 0,3 & 0,5 & 0,7 & 1,0 & 1,4 \\
1200 & 0,0 & 0,3 & 0,5 & 0,7 & 0,8 & 1,1 \\
1400 & 0,0 & 0,3 & 0,5 & 0,6 & 0,8 & 1,0 \\
1600 & 0,0 & 0,4 & 0,5 & 0,6 & 0,8 & 1,0 \\
1800 & 0,0 & 0,2 & 0,4 & 0,5 & 0,6 & 0,9 \\
2000 & 0,0 & 0,2 & 0,4 & 0,5 & 0,6 & 0,7 \\
2200 & 0,0 & 0,2 & 0,2 & 0,3 & 0,5 & 0,6 \\
2400 & 0,0 & 0,2 & 0,3 & 0,3 & 0,4 & 0,5 \\
2600 & 0,0 & 0,2 & 0,3 & 0,4 & 0,5 & 0,4 \\
2800 & 0,0 & 0,3 & 0,3 & 0,5 & 0,6 & 0,3 \\
3000 & 0,0 & 0,4 & 0,5 & 0,6 & 0,7 & 0,4 \\
3200 & 0,0 & 0,4 & 0,5 & 0,6 & 0,6 & 0,3 \\
\hline
\end{tabular}

Tabela 6. Fator de ajuste $f_{d / n p}$ na determinação da PTSF em segmentos com sentido duplo

\begin{tabular}{|c|c|c|c|c|c|c|}
\hline \multirow{2}{*}{$\begin{array}{l}\text { Taxa de flu- } \\
\text { xo nos dois } \\
\text { sentidos } \\
\text { (cpe/h) }\end{array}$} & \multicolumn{6}{|c|}{ Zonas de ultrapassagem proibida (\%) } \\
\hline & 0 & 20 & 40 & 60 & 80 & 100 \\
\hline \multicolumn{7}{|c|}{ Divisão do tráfego $=50 / 50$} \\
\hline $\begin{array}{l}\leq 200 \\
\end{array}$ & 0,0 & 0,9 & 1,4 & 2,1 & 3,0 & 5,8 \\
\hline 400 & 0,0 & 0,8 & 1,5 & 2,4 & 3,9 & 6,1 \\
\hline 600 & 0,0 & 1,0 & 1,8 & 3,0 & 3,8 & 5,3 \\
\hline 800 & 0,0 & 0,9 & 1,5 & 2,1 & 3,1 & 4,6 \\
\hline 1400 & 0,0 & 0,5 & 0,9 & 1,2 & 1,6 & 2,2 \\
\hline 2000 & 0,0 & 0,3 & 0,5 & 0,5 & 0,7 & 1,0 \\
\hline 2600 & 0,0 & 0,2 & 0,2 & 0,3 & 0,4 & 0,5 \\
\hline 3200 & 0,0 & 0,1 & 0,0 & 0,1 & 0,1 & 0,3 \\
\hline \multicolumn{7}{|c|}{ Divisão do tráfego $=60 / 40$} \\
\hline$\leq 200$ & 0,4 & 1,8 & 2,6 & 3,2 & 4,3 & 8,1 \\
\hline 400 & 0,7 & 1,9 & 3,0 & 4,2 & 5,4 & 8,0 \\
\hline 600 & 0,6 & 0,8 & 1,4 & 2,3 & 3,2 & 4,9 \\
\hline 800 & 0,5 & 1,9 & 2,4 & 3,0 & 3,7 & 4,8 \\
\hline 1400 & 0,1 & 0,7 & 0,8 & 1,2 & 1,4 & 2,0 \\
\hline 2000 & 0,0 & 0,9 & 1,0 & 1,3 & 1,5 & 1,7 \\
\hline$\geq 2600$ & 0,0 & 0,5 & 0,6 & 0,9 & 0,8 & 0,8 \\
\hline \multicolumn{7}{|c|}{ Divisão do tráfego $=70 / 30$} \\
\hline $\begin{array}{l}\leq 200 \\
\end{array}$ & 2,0 & 3,3 & 4,2 & 5,0 & 5,8 & 9,3 \\
\hline 400 & 2,2 & 3,5 & 3,9 & 5,1 & 6,8 & 9,5 \\
\hline 600 & 1,4 & 2,6 & 3,6 & 4,3 & 5,8 & 7,7 \\
\hline 800 & 0,3 & 1,2 & 1,8 & 2,5 & 3,5 & 4,7 \\
\hline 1400 & 0,8 & 1,5 & 1,8 & 2,1 & 2,7 & 3,4 \\
\hline$\geq 2000$ & 0,8 & 1,2 & 1,4 & 1,7 & 2,0 & 2,1 \\
\hline \multicolumn{7}{|c|}{ Divisão do tráfego $=80 / 20$} \\
\hline$\leq 200$ & 3,5 & 4,4 & 5,8 & 6,6 & 7,8 & 12,6 \\
\hline 400 & 4,4 & 6,0 & 7,7 & 8,7 & 10,2 & 13,5 \\
\hline 600 & 3,4 & 5,4 & 6,1 & 7,3 & 8,1 & 10,7 \\
\hline 800 & 1,4 & 3,0 & 3,7 & 4,4 & 5,7 & 7,5 \\
\hline 1400 & 0,0 & 2,8 & 3,0 & 3,6 & 4,3 & 4,9 \\
\hline$\geq 2000$ & 0,0 & 1,5 & 1,9 & 2,3 & 2,8 & 2,7 \\
\hline \multicolumn{7}{|c|}{ Divisão do tráfego $=90 / 10$} \\
\hline$\leq 200$ & 9,5 & 10,6 & 11,7 & 12,7 & 13,6 & 17,2 \\
\hline 400 & 8,0 & 10,1 & 11,2 & 11,9 & 13,5 & 16,8 \\
\hline 600 & 5,8 & 7,7 & 8,5 & 9,7 & 11,0 & 13,8 \\
\hline 800 & 3,0 & 4,7 & 5,7 & 6,7 & 7,9 & 9,8 \\
\hline$\geq 1400$ & 1,4 & 2,7 & 3,7 & 4,5 & 5,3 & 6,5 \\
\hline
\end{tabular}

Tabela 7. Fator de ajuste $f_{n p}$ na determinação da ATS em segmentos em sentido único

\begin{tabular}{|c|c|c|c|c|c|}
\hline \multirow{3}{*}{$\begin{array}{l}\text { Taxa de fluxo } \\
\text { no sentido } \\
\text { oposto (cpe/h) }\end{array}$} & \multicolumn{5}{|c|}{ Zonas de ultrapassagem proibida (\%) } \\
\hline & $\leq 20$ & 40 & 60 & 80 & 100 \\
\hline & \multicolumn{5}{|c|}{$F F S=110 \mathrm{~km} / \mathrm{h}$} \\
\hline$\leq 100$ & 0,1 & 0,3 & 0,5 & 0,7 & 1,3 \\
\hline 200 & 1,0 & 1,2 & 1,5 & 1,9 & 2,7 \\
\hline 400 & 1,1 & 1,3 & 1,5 & 1,9 & 2,5 \\
\hline 600 & 0,6 & 0,7 & 0,9 & 1,2 & 1,5 \\
\hline 800 & 0,2 & 0,3 & 0,4 & 0,6 & 0,8 \\
\hline 1000 & 0,5 & 0,6 & 0,7 & 0,9 & 1,1 \\
\hline 1200 & 0,5 & 0,5 & 0,6 & 0,9 & 1,0 \\
\hline 1400 & 0,5 & 0,4 & 0,5 & 0,8 & 0,9 \\
\hline \multirow[t]{2}{*}{$\geq 1600$} & 0,3 & 0,2 & 0,3 & 0,6 & 0,7 \\
\hline & \multicolumn{5}{|c|}{$F F S=100 \mathrm{~km} / \mathrm{h}$} \\
\hline$\leq 100$ & 0,2 & 0,5 & 0,7 & 0,9 & 1,8 \\
\hline 200 & 1,1 & 1,4 & 1,8 & 2,2 & 3,2 \\
\hline 400 & 0,9 & 1,3 & 1,7 & 2,1 & 2,8 \\
\hline 600 & 0,7 & 1,0 & 1,2 & 1,6 & 2,1 \\
\hline 800 & 0,4 & 0,6 & 0,7 & 1,0 & 1,3 \\
\hline 1000 & 0,6 & 0,9 & 1,2 & 1,4 & 1,7 \\
\hline 1200 & 0,6 & 0,8 & 1,1 & 1,3 & 1,6 \\
\hline 1400 & 0,6 & 0,7 & 1,0 & 1,2 & 1,4 \\
\hline \multirow[t]{2}{*}{$\geq 1600$} & 0,4 & 0,5 & 0,8 & 1,0 & 1,2 \\
\hline & \multicolumn{5}{|c|}{$F F S=90 \mathrm{~km} / \mathrm{h}$} \\
\hline$\leq 100$ & 0,2 & $\overline{0,6}$ & 0,9 & 1,2 & 2,3 \\
\hline 200 & 1,3 & 1,7 & 2,2 & 2,8 & 4,1 \\
\hline 400 & 1,0 & $1,, 5$ & 1,9 & 2,5 & 3,4 \\
\hline 600 & 0,9 & 1,2 & 1,7 & 2,0 & 2,7 \\
\hline 800 & 0,6 & 1,0 & 1,2 & 1,5 & 2,1 \\
\hline 1000 & 1,0 & 1,3 & 1,6 & 2,0 & 2,4 \\
\hline 1200 & 0,6 & 0,8 & 1,2 & 1,5 & 1,8 \\
\hline 1400 & 0,2 & 0,4 & 0,7 & 1,0 & 1,2 \\
\hline \multirow[t]{2}{*}{$\geq 1600$} & 0,2 & 0,2 & 0,5 & 0,8 & 1,0 \\
\hline & \multicolumn{5}{|c|}{$F F S=80 \mathrm{~km} / \mathrm{h}$} \\
\hline$\leq 100$ & 0,9 & 1,5 & 1,8 & 2,3 & 3,8 \\
\hline 200 & 1,2 & 1,8 & 2,4 & 3,0 & 4,7 \\
\hline 400 & 1,3 & 1,9 & 2,5 & 3,2 & 4,5 \\
\hline 600 & 1,0 & 1,6 & 2,2 & 2,6 & 3,5 \\
\hline 800 & 0,8 & 1,2 & 1,5 & 2,1 & 2,7 \\
\hline 1000 & 0,7 & 1,2 & 1,6 & 2,1 & 2,5 \\
\hline 1200 & 0,8 & 1,3 & 1,6 & 1,9 & 2,2 \\
\hline 1400 & 0,9 & 1,4 & 1,6 & 1,8 & 1,8 \\
\hline \multirow{2}{*}{$\geq 1600$} & 0,7 & 1,2 & 1,4 & 1,6 & 1,6 \\
\hline & \multicolumn{5}{|c|}{$F F S=70 \mathrm{~km} / \mathrm{h}$} \\
\hline$\leq 100$ & 0,9 & 1,5 & 2,1 & 2,7 & 4,7 \\
\hline 200 & 1,6 & 2,3 & 3,2 & 4,0 & 6,1 \\
\hline 400 & 1,4 & 2,1 & 2,8 & 3,7 & 5,5 \\
\hline 600 & 1,4 & 2,1 & 2,6 & 3,3 & 4,5 \\
\hline 800 & 0,9 & 1,4 & 1,9 & 2,5 & 3,3 \\
\hline 1000 & 1,0 & 1,6 & 2,1 & 2,6 & 3,0 \\
\hline 1200 & 1,9 & 2,4 & 2,8 & 3,1 & 3,1 \\
\hline 1400 & 1,0 & 1,2 & 1,5 & 1,7 & 1,6 \\
\hline$\geq 1600$ & 0,0 & 0,0 & 0,2 & 0,3 & 0,1 \\
\hline
\end{tabular}


Tabela 8. Fator de ajuste $f_{n p}$ na determinação da PTSF em segmentos em sentido único

\begin{tabular}{|c|c|c|c|c|c|}
\hline \multirow{3}{*}{$\begin{array}{l}\text { Taxa de fluxo } \\
\text { no sentido } \\
\text { oposto (cpe/h) }\end{array}$} & \multicolumn{5}{|c|}{ Zonas de ultrapassagem proibida (\%) } \\
\hline & $<20$ & 40 & 60 & 80 & 100 \\
\hline & \multicolumn{5}{|c|}{$F F S=110 \mathrm{~km} / \mathrm{h}$} \\
\hline$\leq 100$ & 0,2 & 0,5 & 1,2 & 2,4 & 4,5 \\
\hline 200 & 1,4 & 2,2 & 3,1 & 4,4 & 6,5 \\
\hline 400 & 0,3 & 1,0 & 1,5 & 2,5 & 4,0 \\
\hline 600 & 0,1 & 0,4 & 0,7 & 1,1 & 2,2 \\
\hline 800 & 0,0 & 0,1 & 0,2 & 0,6 & 1,0 \\
\hline 1000 & 1,1 & 1,2 & 1,5 & 1,6 & 1,9 \\
\hline 1200 & 0,7 & 0,8 & 0,9 & 1,1 & 1,4 \\
\hline 1400 & 0,5 & 0,6 & 0,6 & 0,7 & 0,8 \\
\hline \multirow[t]{2}{*}{$\geq 1600$} & 0,2 & 0,3 & 0,3 & 0,4 & 0,5 \\
\hline & \multicolumn{5}{|c|}{$F F S=100 \mathrm{~km} / \mathrm{h}$} \\
\hline$\leq 100$ & 0,0 & 1,4 & 2,0 & 3,0 & 5,9 \\
\hline 200 & 1,4 & 2,3 & 3,4 & 5,0 & 8,3 \\
\hline 400 & 0,8 & 0,9 & 1,6 & 3,0 & 4,8 \\
\hline 600 & 0,1 & 0,8 & 1,3 & 1,9 & 3,1 \\
\hline 800 & 0,0 & 0,3 & 0,6 & 1,0 & 1,6 \\
\hline 1000 & 0,9 & 1,2 & 1,5 & 1,8 & 2,3 \\
\hline 1200 & 0,5 & 0,7 & 0,8 & 1,1 & 1,4 \\
\hline 1400 & 0,7 & 0,7 & 0,8 & 0,7 & 0,9 \\
\hline \multirow[t]{2}{*}{$\geq 1600$} & 0,4 & 0,4 & 0,5 & 0,4 & 0,6 \\
\hline & \multicolumn{5}{|c|}{$F F S=90 \mathrm{~km} / \mathrm{h}$} \\
\hline$\leq 100$ & 0,4 & 1,8 & 2,8 & 3,8 & 7,4 \\
\hline 200 & 1,9 & 3,0 & 4,0 & 5,8 & 9,8 \\
\hline 400 & 0,2 & 1,1 & 2,1 & 3,5 & 5,9 \\
\hline 600 & 0,1 & 0,7 & 1,2 & 2,2 & 3,8 \\
\hline 800 & 0,1 & 0,6 & 0,9 & 1,4 & 2,3 \\
\hline 1000 & 0,0 & 0,2 & 0,5 & 1,0 & 1,6 \\
\hline 1200 & 0,0 & 0,1 & 0,3 & 0,6 & 0,9 \\
\hline 1400 & 0,1 & 0,0 & 0,1 & 0,2 & 0,1 \\
\hline \multirow[t]{2}{*}{$\geq 1600$} & 0,1 & 0,0 & 0,1 & 0,2 & 0,1 \\
\hline & \multicolumn{5}{|c|}{$F F S=80 \mathrm{~km} / \mathrm{h}$} \\
\hline$\leq 100$ & 3,6 & 5,3 & 6,7 & 8,2 & 12,2 \\
\hline 200 & 2,4 & 3,6 & 5,2 & 7,2 & 12,3 \\
\hline 400 & 0,5 & 1,6 & 2,9 & 4,6 & 7,5 \\
\hline 600 & 0,6 & 0,3 & 1,3 & 2,4 & 4,7 \\
\hline 800 & 0,7 & 0,9 & 1,6 & 2,1 & 3,2 \\
\hline 1000 & 0,4 & 0,8 & 0,8 & 0,9 & 1,4 \\
\hline 1200 & 0,2 & 0,5 & 0,5 & 0,6 & 1,2 \\
\hline 1400 & 0,1 & 0,2 & 0,2 & 0,3 & 1,0 \\
\hline \multirow[t]{2}{*}{$\geq 1600$} & 0,1 & 0,1 & 0,1 & 0,2 & 0,5 \\
\hline & \multicolumn{5}{|c|}{$F F S=70 \mathrm{~km} / \mathrm{h}$} \\
\hline$\leq 100$ & 4,5 & 6,2 & 7,8 & 9,7 & 15,6 \\
\hline 200 & 3,8 & 5,5 & 7,8 & 10,2 & 16,2 \\
\hline 400 & 0,5 & 0,9 & 2,5 & 4,4 & 8,1 \\
\hline 600 & 0,5 & 0,3 & 1,0 & 2,3 & 4,2 \\
\hline 800 & 0,5 & 0,9 & 1,4 & 2,0 & 2,8 \\
\hline 1000 & 0,5 & 0,5 & 0,7 & 0,4 & 0,3 \\
\hline 1200 & 0,3 & 0,6 & 0,6 & 0,3 & 0,2 \\
\hline 1400 & 0,2 & 0,6 & 0,5 & 0,3 & 0,1 \\
\hline$\geq 1600$ & 0,2 & 0,1 & 0,1 & 0,1 & 0,1 \\
\hline
\end{tabular}

\subsection{Rampas específicas}

O método de análise de rampas específicas é voltado para trechos com extensão acima de $0,4 \mathrm{~km}$ e que possuem aclives maiores ou iguais a $3 \%$, com ou sem faixas adicionais (de subida e de ultrapassagem). A determinação de $F F S$, da taxa de fluxo e das relações fundamentais segue o mesmo procedimento apresentado nas seções 3.1.1 a 3.1.3, exceto os fatores de ajuste usados, como mostrado nas Tabelas 9 a 14 para locais sem faixas adicionais. No caso da Tabela 10 , para estimação de $P T S F_{d}$, os fatores de ajuste $f_{G}$ são os mesmos sugeridos pelo HCM2000. Isso se deve ao fato de que os valores de $P T S F_{d}$ fornecidos pelo simulador TWOPAS durante a adaptação mostraram-se inconsistentes. Ao contrário do que era esperado, os valores de $P T S F_{d}$ decresceram à medida que a declividade e o comprimento da rampa aumentaram.

Sabe-se que o efeito da presença de caminhões na corrente de tráfego varia em função de fatores como o relevo, o fluxo do tráfego e a porcentagem de caminhões. Entretanto, durante a elaboração do Capítulo 20 do HCM2000, não foi possível mostrar que a porcentagem de caminhões tinha um efeito significativo que justificasse a inclusão na determinação de $E_{T}$. Para este trabalho, foi proposto uma melhoria a partir da fixação de um conjunto de caminhões típicos para as rodovias de pista simples brasileiras e da variação da porcentagem de caminhões no fluxo total de tráfego, $20 \%, 30 \%$ e $40 \%$, porcentagens compatíveis com os observados em rodovias brasileiras. Os valores encontrados para $E_{T}$ usando essa estratificação podem ser visualizados nas Tabelas 11 e 12 .

Tabela 9. Fator de ajuste $f_{G}$ na determinação de $A T S_{d}$ em rampas específicas

\begin{tabular}{|c|c|c|c|c|}
\hline \multirow{2}{*}{$\begin{array}{l}\text { Declividade } \\
\text { (\%) }\end{array}$} & \multirow{2}{*}{$\begin{array}{l}L \\
(k m)\end{array}$} & \multicolumn{2}{|c|}{$\begin{array}{l}\text { Taxa de fluxo em } \\
\text { sentido único (cpe/h) }\end{array}$} & \multirow[b]{2}{*}{$>600$} \\
\hline & & $0-300$ & $>300-600$ & \\
\hline & 0,4 & 0,83 & 0,93 & 1,00 \\
\hline & 0,8 & 0,80 & 0,94 & 1,00 \\
\hline & 1,2 & 0,80 & 0,95 & 1,00 \\
\hline & 1,6 & 0,80 & 0,94 & 0,99 \\
\hline & 2,4 & 0,77 & 0,94 & 0,99 \\
\hline & 3,2 & 0,74 & 0,90 & 0,98 \\
\hline & 4,8 & 0,71 & 0,88 & 0,98 \\
\hline \multirow[t]{8}{*}{$\geq 3,0<3,5$} & $\geq 6,4$ & 0,69 & 0,87 & 0,97 \\
\hline & 0,4 & 0,82 & 0,93 & 1,00 \\
\hline & 0,8 & 0,74 & 0,92 & 0,99 \\
\hline & 1,2 & 0,73 & 0,91 & 0,99 \\
\hline & 1,6 & 0,68 & 0,89 & 0,98 \\
\hline & 2,4 & 0,63 & 0,87 & 0,98 \\
\hline & 3,2 & 0,57 & 0,82 & 0,97 \\
\hline & 4,8 & 0,53 & 0,81 & 0,97 \\
\hline \multirow[t]{8}{*}{$\geq 3,5<4,5$} & $\geq 6,4$ & 0,50 & 0,77 & 0,96 \\
\hline & 0,4 & 0,77 & 0,92 & 0,99 \\
\hline & 0,8 & 0,66 & 0,87 & 0,98 \\
\hline & 1,2 & 0,56 & 0,80 & 0,95 \\
\hline & 1,6 & 0,51 & 0,75 & 0,92 \\
\hline & 2,4 & 0,43 & 0,70 & 0,91 \\
\hline & 3,2 & 0,38 & 0,63 & 0,88 \\
\hline & 4,8 & 0,33 & 0,58 & 0,87 \\
\hline \multirow[t]{8}{*}{$\geq 4,5<5,5$} & $\geq 6,4$ & 0,30 & 0,55 & 0,86 \\
\hline & 0,4 & 0,68 & 0,87 & 0,98 \\
\hline & 0,8 & 0,53 & 0,74 & 0,91 \\
\hline & 1,2 & 0,42 & 0,64 & 0,83 \\
\hline & 1,6 & 0,35 & 0,56 & 0,77 \\
\hline & 2,4 & 0,27 & 0,47 & 0,70 \\
\hline & 3,2 & 0,24 & 0,42 & 0,66 \\
\hline & 4,8 & 0,20 & 0,37 & 0,62 \\
\hline \multirow[t]{8}{*}{$\geq 5,5<6,5$} & $\geq 6,4$ & 0,19 & 0,35 & 0,60 \\
\hline & 0,4 & 0,59 & 0,81 & 0,96 \\
\hline & 0,8 & 0,41 & 0,61 & 0,80 \\
\hline & 1,2 & 0,30 & 0,48 & 0,68 \\
\hline & 1,6 & 0,24 & 0,41 & 0,61 \\
\hline & 2,4 & 0,19 & 0,33 & 0,52 \\
\hline & 3,2 & 0,16 & 0,29 & 0,48 \\
\hline & 4,8 & 0,14 & 0,25 & 0,44 \\
\hline$\geq 6,5$ & $\geq 6,4$ & 0,13 & 0,23 & 0,42 \\
\hline
\end{tabular}


Tabela 10. Fator de ajuste $f_{G}$ na determinação de $P T S F_{d}$ em rampas específicas

\begin{tabular}{|c|c|c|c|c|}
\hline \multirow{2}{*}{$\begin{array}{l}\text { Declividade } \\
\text { (\%) }\end{array}$} & \multirow{2}{*}{$\begin{array}{l}L \\
(k m)\end{array}$} & \multicolumn{2}{|c|}{$\begin{array}{l}\text { Taxa de fluxo em } \\
\text { sentido único }(\text { cpe/h) }\end{array}$} & \multirow[b]{2}{*}{$>600$} \\
\hline & & $0-300$ & $>300-600$ & \\
\hline & 0,4 & 1,00 & 0,92 & 0,92 \\
\hline & 0,8 & 1,00 & 0,93 & 0,93 \\
\hline & 1,2 & 1,00 & 0,93 & 0,93 \\
\hline & 1,6 & 1,00 & 0,93 & 0,93 \\
\hline & 2,4 & 1,00 & 0,94 & 0,94 \\
\hline & 3,2 & 1,00 & 0,95 & 0,95 \\
\hline & 4,8 & 1,00 & 0,97 & 0,96 \\
\hline \multirow[t]{8}{*}{$\geq 3,0<3,5$} & $\geq 6,4$ & 1,00 & 1,00 & 0,97 \\
\hline & 0,4 & 1,00 & 0,94 & 0,92 \\
\hline & 0,8 & 1,00 & 0,97 & 0,96 \\
\hline & 1,2 & 1,00 & 0,97 & 0,96 \\
\hline & 1,6 & 1,00 & 0,97 & 0,97 \\
\hline & 2,4 & 1,00 & 0,97 & 0,97 \\
\hline & 3,2 & 1,00 & 0,98 & 0,98 \\
\hline & 4,8 & 1,00 & 1,00 & 1,00 \\
\hline \multirow[t]{8}{*}{$\geq 3,5<4,5$} & $\geq 6,4$ & 1,00 & 1,00 & 1,00 \\
\hline & 0,4 & 1,00 & 1,00 & 0,97 \\
\hline & 0,8 & 1,00 & 1,00 & 1,00 \\
\hline & 1,2 & 1,00 & 1,00 & 1,00 \\
\hline & 1,6 & 1,00 & 1,00 & 1,00 \\
\hline & 2,4 & 1,00 & 1,00 & 1,00 \\
\hline & 3,2 & 1,00 & 1,00 & 1,00 \\
\hline & 4,8 & 1,00 & 1,00 & 1,00 \\
\hline \multirow[t]{8}{*}{$\geq 4,5<5,5$} & $\geq 6,4$ & 1,00 & 1,00 & 1,00 \\
\hline & 0,4 & 1,00 & 1,00 & 1,00 \\
\hline & 0,8 & 1,00 & 1,00 & 1,00 \\
\hline & 1,2 & 1,00 & 1,00 & 1,00 \\
\hline & 1,6 & 1,00 & 1,00 & 1,00 \\
\hline & 2,4 & 1,00 & 1,00 & 1,00 \\
\hline & 3,2 & 1,00 & 1,00 & 1,00 \\
\hline & 4,8 & 1,00 & 1,00 & 1,00 \\
\hline \multirow[t]{8}{*}{$\geq 5,5<6,5$} & $\geq 6,4$ & 1,00 & 1,00 & 1,00 \\
\hline & 0,4 & 1,00 & 1,00 & 1,00 \\
\hline & 0,8 & 1,00 & 1,00 & 1,00 \\
\hline & 1,2 & 1,00 & 1,00 & 1,00 \\
\hline & 1,6 & 1,00 & 1,00 & 1,00 \\
\hline & 2,4 & 1,00 & 1,00 & 1,00 \\
\hline & 3,2 & 1,00 & 1,00 & 1,00 \\
\hline & 4,8 & 1,00 & 1,00 & 1,00 \\
\hline$\geq 6,5$ & $\geq 6,4$ & 1,00 & 1,00 & 1,00 \\
\hline
\end{tabular}

\subsection{Segmentos em sentido único com faixas adicionais}

Inicialmente, deve-se calcular as medidas de desempenho $A T S_{d}$ e $P T S F_{d}$, conforme a seção 3.1. Devem ser determinados na análise de segmentos com faixas adicionais, em quilômetros: o comprimento total do trecho analisado $\left(L_{t}\right)$, o tamanho do trecho antes do início da faixa de ultrapassagem $\left(L_{u}\right)$, o comprimento do trecho após os efeitos da faixa de ultrapassagem terem cessado $\left(L_{d}\right)$, a faixa adicional propriamente dita $\left(L_{p l}\right)$ e o trecho após o término da faixa de ultrapassagem, dentro do comprimento efetivo da faixa de ultrapassagem $\left(L_{d e}\right)$. Para segmentos com faixas adicionais em subidas, os parâmetros $L_{u}, L_{d e}$ e $L_{d}$ são iguais a zero e $L_{p l}$ e $L_{t}$ possuem o mesmo valor. Por fim, calculam-se os valores das medidas de desempenho em faixas adicionais
$\left(A S T_{p l}\right.$ e $\left.P T S F_{p l}\right)$ de acordo com as Equações 9 e $10 . \mathrm{O}$ fator de ajuste para o efeito das faixas adicionais $\left(f_{p l}\right)$ pode ser obtido por meio das Tabelas 15 e 17 .

$$
\begin{gathered}
A T S_{p l}=\frac{A T S_{d} L_{t}}{L_{u}+L_{d}+\frac{L_{p l}}{f_{p l}}+\frac{2 L_{d e}}{1+f_{p l}}} \\
P T S F_{p l}=\frac{P T S F_{d}\left[L_{u}+L_{d}+f_{p l} L_{p l}+\left(\frac{1+f_{p l}}{2}\right) L_{d e}\right]}{L_{t}}
\end{gathered}
$$

\section{ESTIMAÇÃO DO NÍVEL DE SERVIÇO COM A ADAPTAÇÃO PROPOSTA}

Para avaliar a acurácia da metodologia proposta, foram comparados os níveis de serviço observados em campo com a adaptação desenvolvida e com o HCM2000 sem alterações. Essa verificação foi realizada em um trabalho anterior (Utimura et al., 2007) para trechos sem faixas adicionais. Nesse caso, foi realizada uma análise em dois sentidos com cada hora observada sendo considerada uma corrente de tráfego distinta. A medida de desempenho $A T S_{d}$ foi obtida diretamente da corrente de tráfego, o que é impossível para $P T S F_{d}$, estimada usando o simulador TWOPAS recalibrado para cada trecho estudado. A velocidade de fluxo livre em um sentido $\left(F F S_{d}\right)$ foi calculada usando a Equação 1 e a Equação 20-1 do HCM2000. Os resultados dos níveis de serviço, mostrados na Tabela 18 , indicam que a adaptação proposta tende a ser mais confiável, com um índice de acertos próximo de $85 \%$.

\section{RECOMENDAÇÕES}

A adaptação apresentada neste trabalho, provenientes de duas teses de doutorado (Egami, 2006; Mon-Ma, 2008) faz parte de uma linha de pesquisa que tem sido desenvolvida com o intuito de obter um manual de capacidade viária para o Brasil. Os resultados provenientes de um outro trabalho (Utimura et al., 2007) indicam que, ao se optar por uma adaptação do método do HCM2000, as estimativas dos níveis de serviço de estradas paulistas tendem a ser bem mais confiáveis, com um índice de acertos acima de $80 \%$. Essa avaliação foi feita para rodovias sem faixas adicionais, mas, em projetos futuros, um estudo semelhante pode ser realizado para segmentos de rodovias com faixas adicionais.

Recomenda-se ainda obter uma adaptação que, a priori, não tenha que seguir a mesma estrutura do HCM2000. Além disso, há necessidade de se obter conjuntos de dados mais representativos de toda a gama de condições operacionais, e não apenas para tráfego com fluxo baixo. Com isso, poderão ser realizados estudos mais detalhados sobre:

- As relações entre o fluxo de tráfego e as medidas de desempenho; a relação fluxo-velocidade do HCM2000, por exemplo, tem um formato linear, que pode não ser o mais adequado para as condições das estradas brasileiras, como indica outro artigo (Bessa Jr. e Setti, 2011);

- Os fatores de ajuste para condições não básicas; alguns fatores adotados pelo HCM2000, como aqueles voltados para a largura da faixa e do acostamento e 
Tabela 11. Equivalente veicular $E_{T}$ na determinação de $A T S_{d}$ em rampas específicas

\begin{tabular}{|c|c|c|c|c|c|c|c|c|c|c|}
\hline \multirow{4}{*}{$\begin{array}{l}\text { Declividade } \\
\text { (\%) }\end{array}$} & \multirow{4}{*}{$\begin{array}{l}L \\
(\mathrm{~km})\end{array}$} & \multirow{2}{*}{\multicolumn{3}{|c|}{$\begin{array}{l}\text { Taxa de fluxo em } \\
\text { sentido único (cpe/h) } \\
0-300\end{array}$}} & \multirow{3}{*}{\multicolumn{3}{|c|}{$\begin{array}{l}\text { Taxa de fluxo em } \\
\text { sentido único (cpe/h) } \\
300-600 \\
\text { Caminhões (\%) }\end{array}$}} & \multirow{3}{*}{\multicolumn{3}{|c|}{$\begin{array}{l}\text { Taxa de fluxo em } \\
\text { sentido único (cpe/h) } \\
>600\end{array}$}} \\
\hline & & & & & & & & & & \\
\hline & & \multicolumn{3}{|c|}{ Caminhões (\%) } & & & & & & Caminhões (\%) \\
\hline & & 20 & 30 & 40 & 20 & 30 & 40 & 20 & 30 & 40 \\
\hline & 0,4 & 3,6 & 3,5 & 3,1 & 2,8 & 2,8 & 2,7 & 2,3 & 2,3 & 2,2 \\
\hline & 0,8 & 5,0 & 4,9 & 4,8 & 3,9 & 3,9 & 3,7 & 3,3 & 3,1 & 2,9 \\
\hline & 1,2 & 6,1 & 6,0 & 5,9 & 5,1 & 4,8 & 4,4 & 4,1 & 3,6 & 3,3 \\
\hline & 1,6 & 7,1 & 7,0 & 6,7 & 6,0 & 5,5 & 5,0 & 4,9 & 4,2 & 3,7 \\
\hline & 2,4 & 8,3 & 8,3 & 7,8 & 7,8 & 6,7 & 5,9 & 6,0 & 4,9 & 4,2 \\
\hline & 3,2 & 9,3 & 9,3 & 8,8 & 8,9 & 7,5 & 6,6 & 6,7 & 5,4 & 4,5 \\
\hline & 4,8 & 10,7 & 10,3 & 9,8 & 10,8 & 8,7 & 7,5 & 7,7 & 6,0 & 5,0 \\
\hline \multirow[t]{8}{*}{$\geq 3,0<3,5$} & $\geq 6,4$ & 11,6 & 11,1 & 10,3 & 11,9 & 9,4 & 8,1 & 8,2 & 6,3 & 5,2 \\
\hline & 0,4 & 4,3 & 4,2 & 4,1 & 3,5 & 3,5 & 3,4 & 3,0 & 2,8 & 2,7 \\
\hline & 0,8 & 6,8 & 6,6 & 6,3 & 5,5 & 5,2 & 4,7 & 4,7 & 4,1 & 3,7 \\
\hline & 1,2 & 8,4 & 8,2 & 7,6 & 7,4 & 6,7 & 5,9 & 6,2 & 5,1 & 4,4 \\
\hline & 1,6 & 9,5 & 9,2 & 8,4 & 9,2 & 7,8 & 6,8 & 7,2 & 5,8 & 4,9 \\
\hline & 2,4 & 10,8 & 10,3 & 9,5 & 11,4 & 9,4 & 7,9 & 8,7 & 6,7 & 5,5 \\
\hline & 3,2 & 11,8 & 11,1 & 10,2 & 12,9 & 10,3 & 8,7 & 9,6 & 7,3 & 6,0 \\
\hline & 4,8 & 13,6 & 12,3 & 10,9 & 14,5 & 11,4 & 9,6 & 10,6 & 7,9 & 6,4 \\
\hline \multirow[t]{8}{*}{$\geq 3,5<4,5$} & $\geq 6,4$ & 14,8 & 13,2 & 11,3 & 16,1 & 12,4 & 10,4 & 11,2 & 8,3 & 6,7 \\
\hline & 0,4 & 5,2 & 5,1 & 5,0 & 4,4 & 4,3 & 4,0 & 3,8 & 3,5 & 3,2 \\
\hline & 0,8 & 8,9 & 8,4 & 7,7 & 7,8 & 6,9 & 6,1 & 6,4 & 5,4 & 4,7 \\
\hline & 1,2 & 10,4 & 9,8 & 8,9 & 10,2 & 8,7 & 7,4 & 8,3 & 6,5 & 5,5 \\
\hline & 1,6 & 11,2 & 10,7 & 9,7 & 11,8 & 9,7 & 8,2 & 9,5 & 7,3 & 6,0 \\
\hline & 2,4 & 12,2 & 11,4 & 10,4 & 13,8 & 10,9 & 9,2 & 10,9 & 8,2 & 6,6 \\
\hline & 3,2 & 13,1 & 11,9 & 10,9 & 15,5 & 11,9 & 9,8 & 11,8 & 8,8 & 7,1 \\
\hline & 4,8 & 14,7 & 13,0 & 11,4 & 17,0 & 13,0 & 10,7 & 12,8 & 9,4 & 7,5 \\
\hline \multirow[t]{8}{*}{$\geq 4,5<5,5$} & $\geq 6,4$ & 15,6 & 13,8 & 11,9 & 18,2 & 13,8 & 11,2 & 13,4 & 9,8 & 7,8 \\
\hline & 0,4 & 6,2 & 6,1 & 5,9 & 5,8 & 5,5 & 5,0 & 5,0 & 4,4 & 4,0 \\
\hline & 0,8 & 9,9 & 9,8 & 8,7 & 10,2 & 8,6 & 7,5 & 8,3 & 6,7 & 5,7 \\
\hline & 1,2 & 11,0 & 10,7 & 9,8 & 12,5 & 10,1 & 8,6 & 10,1 & 7,8 & 6,5 \\
\hline & 1,6 & 11,6 & 11,3 & 10,2 & 13,9 & 11,0 & 9,1 & 11,2 & 8,5 & 6,9 \\
\hline & 2,4 & 12,6 & 11,7 & 10,6 & 15,5 & 11,9 & 9,9 & 12,5 & 9,2 & 7,4 \\
\hline & 3,2 & 13,6 & 12,0 & 11,0 & 16,3 & 12,4 & 10,1 & 13,1 & 9,6 & 7,7 \\
\hline & 4,8 & 14,8 & 13,0 & 11,7 & 17,5 & 13,2 & 10,8 & 14,0 & 10,2 & 8,1 \\
\hline \multirow[t]{8}{*}{$\geq 5,5<6,5$} & $\geq 6,4$ & 15,7 & 13,8 & 12,2 & 18,7 & 13,9 & 11,2 & 14,5 & 10,4 & 8,3 \\
\hline & 0,4 & 8,2 & 7,7 & 7,2 & 7,5 & 6,8 & 6,1 & 6,6 & 5,7 & 5,1 \\
\hline & 0,8 & 11,2 & 10,9 & 9,4 & 12,3 & 10,1 & 8,5 & 10,1 & 7,9 & 6,6 \\
\hline & 1,2 & 12,3 & 11,4 & 10,3 & 14,0 & 11,0 & 9,2 & 11,5 & 8,7 & 7,1 \\
\hline & 1,6 & 12,6 & 11,7 & 10,6 & 15,0 & 11,8 & 9,6 & 12,4 & 9,3 & 7,5 \\
\hline & 2,4 & 13,2 & 12,0 & 11,0 & 15,9 & 12,2 & 10,1 & 13,1 & 9,6 & 7,7 \\
\hline & 3,2 & 13,8 & 12,3 & 11,4 & 16,6 & 12,6 & 10,3 & 13,5 & 9,9 & 7,8 \\
\hline & 4,8 & 14,9 & 13,0 & 11,8 & 17,5 & 13,4 & 10,8 & 14,1 & 10,3 & 8,1 \\
\hline$\geq 6,5$ & $\geq 6,4$ & 15,8 & 13,8 & 12,2 & 18,7 & 14,0 & 11,2 & 14,5 & 10,4 & 8,3 \\
\hline
\end{tabular}

para a densidade de pontos de acesso, não são considerados por outros manuais de capacidade viária (FGSV, 2005); e

- As medidas de desempenho mais adequadas para refletir a qualidade de serviço; a PTSF, sugerida pelo HCM, é impossível de ser obtida diretamente da observação do tráfego; sendo assim, recomenda-se estudar outras medidas de desempenho que não possuam esse tipo de problema.

\section{AGRADECIMENTOS}

Os autores agradecem o apoio da FAPESP e do CNPq, pelo suporte financeiro sob a forma de bolsas de doutorado e de produtividade em pesquisa.

\section{REFERÊNCIAS BIBLIOGRÁFICAS}

ARTESP (2002) Relatório $n^{o}$. 42: Procedimentos Práticos para Cálculo da Capacidade Viária e Determinação do Nível de Serviço de Operação. Empresa de Apoio e Gerenciamento (EAG-ARTESP), Setor de Operações.
Bessa Jr., J. E.; J. R. Setti (2011) Derivation of ATS and PTSF functions for two-lane, rural highways in Brazil. $6^{\text {th }}$ International Symposium on Highway Capacity and Quality of Service, Estocolmo, Proceedings..., Procedia - Social and Behavioral Sciences, Elsevier Ltd., v. 16, p. 282-292.

Brilon, W.; F. Weiser (2006) Two-lane rural highways: the German experience. Transportation Research Record 1988, TRB, National Research Council, Washington, D.C., p. 38-47.

DNIT (2006) Manual de Estudos de Tráfego. Ministério dos Transportes, Departamento Nacional de Infra-Estrutura de Transportes, Rio de Janeiro, RJ.

Egami, C. Y. (2006) Adaptação do HCM-2000 para determinação do nível de serviço em rodovias de pista simples sem faixas adicionais no Brasil. 240p. Tese (Doutorado) - Escola de Engenharia de São Carlos, Universidade de São Paulo, São Carlos.

Egami, C. Y.; M. L. Mon-Ma; J. R. Setti; L. R. Rilett (2006) Automatic calibration of two-lane highway traffic simulation models using a genetic algorithm. Proceedings of 9th International ConferenceApplications of Advanced Technologies in Transportation. American Society of Civil Engineers - ASCE. p. 510-515. Chicago, Illinois.

FGSV (2005) Handbuch zur Bemessung von Strassenverkehrsanlagen 2001 (HBS, German Highway Capacity Manual 2001). 
Forschungsgesellschaft fur Straßen- und Verkehrswesen (FGSV), Cologne.

Goldberg, D. E. (1989) Genetic algorithms in search, optimization and machine learning, Addison-Wesley, Reading, Massachussets.

Harwood, D. W.; A. D. May; I. B. Anderson; L. Leiman; A. R. Archilla (1999) Capacity and quality of service of two-lane highways. Final Report, NCHRP Project 3-55 (3). MRI, Kansas City, Mo.

Mon-Ma, M. L. (2008) Adaptação do HCM-2000 para rodovias de pista simples com faixas adicionais típicas do Estado de São Paulo. 162p. Tese (Doutorado) - Escola de Engenharia de São Carlos, Universidade de São Paulo, São Carlos.

TCU (2008) Acórdão 2104/2008 Plenário, processo no 016.189/2008-9.

Tribunal de Contas da União. Disponível em:

$<$ http://portal2.tcu.gov.br/portal/page/portal/TCU/imprensa/

noticias/detalhes_noticias?noticia $=777746>\quad$ (Acesso em: 7/7/2009).

TRB (2000) Highway Capacity Manual 2000. Transportation Research Board. Washington D.C.

TRB (2010) Highway Capacity Manual 2010. Transportation Research
Board. Washington D.C.

Utimura, F. S.; J. R. Setti; C. Y. Egami e M. L. Mon-Ma (2007) Verificação da acurácia de estimativas do nível de serviço em rodovias de pista simples no estado de São Paulo. XXI ANPET Congresso de Pesquisa e Ensino em Transportes (CD-ROM), Anais..., Rio de Janeiro, RJ, p. 1-8.

Van As, C. (2003) The development of an analysis method for the determination of level of service of two-lane undivided highways in South Africa. Project Summary. South African National Roads Agency Limited.

Tabela 12. Equivalente veicular $E_{T}$ na determinação de $P T S F_{d}$ em rampas específicas

\begin{tabular}{|c|c|c|c|c|c|c|c|c|c|c|}
\hline \multirow{4}{*}{$\begin{array}{l}\text { Declividade } \\
\text { (\%) }\end{array}$} & \multirow{4}{*}{$\begin{array}{l}L \\
(\mathrm{~km})\end{array}$} & \multirow{2}{*}{\multicolumn{3}{|c|}{$\begin{array}{l}\text { Taxa de fluxo em } \\
\text { sentido único } \\
\text { (cpe/h) } \\
0-300\end{array}$}} & \multirow{2}{*}{\multicolumn{3}{|c|}{$\begin{array}{l}\text { Taxa de fluxo em } \\
\text { sentido único } \\
\text { (cpe/h) }\end{array}$}} & \multicolumn{3}{|c|}{$\begin{array}{l}\text { Taxa de fluxo em } \\
\text { sentido único } \\
\text { (cpe/h) }\end{array}$} \\
\hline & & & & & & & & \multicolumn{3}{|c|}{$>600$} \\
\hline & & \multicolumn{3}{|c|}{ Caminhões (\%) } & \multicolumn{3}{|c|}{ Caminhões (\%) } & \multicolumn{3}{|c|}{ Caminhões (\%) } \\
\hline & & 20 & 30 & 40 & 20 & 30 & 40 & 20 & 30 & 40 \\
\hline & 0,4 & 1,0 & 1,0 & 1,0 & 1,0 & 1,0 & 1,0 & 1,0 & 1,0 & 1,0 \\
\hline & 0,8 & 1,1 & 1,0 & 1,0 & 1,0 & 1,0 & 1,0 & 1,0 & 1,0 & 1,0 \\
\hline & 1,2 & 1,1 & 1,0 & 1,0 & 1,0 & 1,0 & 1,0 & 1,0 & 1,0 & 1,0 \\
\hline & 1,6 & 1,1 & 1,1 & 1,0 & 1,0 & 1,0 & 1,0 & 1,0 & 1,0 & 1,0 \\
\hline & 2,4 & 1,2 & 1,1 & 1,1 & 1,0 & 1,0 & 1,0 & 1,0 & 1,0 & 1,0 \\
\hline & 3,2 & 1,2 & 1,2 & 1,1 & 1,1 & 1,0 & 1,0 & 1,0 & 1,0 & 1,0 \\
\hline & 4,8 & 1,3 & 1,2 & 1,2 & 1,1 & 1,0 & 1,0 & 1,0 & 1,0 & 1,0 \\
\hline \multirow[t]{8}{*}{$\geq 3,0<3,5$} & $\geq 6,4$ & 1,4 & 1,3 & 1,3 & 1,2 & 1,1 & 1,0 & 1,0 & 1,0 & 1,0 \\
\hline & 0,4 & 1,1 & 1,0 & 1,0 & 1,0 & 1,0 & 1,0 & 1,0 & 1,0 & 1,0 \\
\hline & 0,8 & 1,2 & 1,1 & 1,0 & 1,0 & 1,0 & 1,0 & 1,0 & 1,0 & 1,0 \\
\hline & 1,2 & 1,2 & 1,1 & 1,1 & 1,0 & 1,0 & 1,0 & 1,0 & 1,0 & 1,0 \\
\hline & 1,6 & 1,3 & 1,2 & 1,1 & 1,1 & 1,0 & 1,0 & 1,0 & 1,0 & 1,0 \\
\hline & 2,4 & 1,3 & 1,3 & 1,2 & 1,1 & 1,1 & 1,0 & 1,0 & 1,0 & 1,0 \\
\hline & 3,2 & 1,4 & 1,3 & 1,3 & 1,2 & 1,1 & 1,0 & 1,0 & 1,0 & 1,0 \\
\hline & 4,8 & 1,5 & 1,4 & 1,4 & 1,2 & 1,1 & 1,1 & 1,0 & 1,0 & 1,0 \\
\hline \multirow[t]{8}{*}{$\geq 3,5<4,5$} & $\geq 6,4$ & 1,6 & 1,5 & 1,5 & 1,2 & 1,1 & 1,1 & 1,0 & 1,0 & 1,0 \\
\hline & 0,4 & 1,1 & 1,0 & 1,0 & 1,0 & 1,0 & 1,0 & 1,0 & 1,0 & 1,0 \\
\hline & 0,8 & 1,2 & 1,2 & 1,1 & 1,0 & 1,0 & 1,0 & 1,0 & 1,0 & 1,0 \\
\hline & 1,2 & 1,4 & 1,3 & 1,2 & 1,1 & 1,0 & 1,0 & 1,0 & 1,0 & 1,0 \\
\hline & 1,6 & 1,4 & 1,3 & 1,3 & 1,1 & 1,1 & 1,0 & 1,0 & 1,0 & 1,0 \\
\hline & 2,4 & 1,5 & 1,4 & 1,3 & 1,2 & 1,1 & 1,0 & 1,0 & 1,0 & 1,0 \\
\hline & 3,2 & 1,6 & 1,5 & 1,4 & 1,2 & 1,1 & 1,1 & 1,0 & 1,0 & 1,0 \\
\hline & 4,8 & 1,7 & 1,6 & 1,5 & 1,3 & 1,2 & 1,1 & 1,0 & 1,0 & 1,0 \\
\hline \multirow[t]{8}{*}{$\geq 4,5<5,5$} & $\geq 6,4$ & 1,9 & 1,7 & 1,6 & 1,3 & 1,2 & 1,1 & 1,0 & 1,0 & 1,0 \\
\hline & 0,4 & 1,1 & 1,0 & 1,0 & 1,0 & 1,0 & 1,0 & 1,0 & 1,0 & 1,0 \\
\hline & 0,8 & 1,3 & 1,3 & 1,2 & 1,1 & 1,1 & 1,0 & 1,0 & 1,0 & 1,0 \\
\hline & 1,2 & 1,4 & 1,4 & 1,3 & 1,2 & 1,1 & 1,0 & 1,0 & 1,0 & 1,0 \\
\hline & 1,6 & 1,5 & 1,4 & 1,4 & 1,2 & 1,1 & 1,0 & 1,0 & 1,0 & 1,0 \\
\hline & 2,4 & 1,6 & 1,5 & 1,5 & 1,2 & 1,1 & 1,1 & 1,0 & 1,0 & 1,0 \\
\hline & 3,2 & 1,7 & 1,6 & 1,5 & 1,3 & 1,2 & 1,1 & 1,0 & 1,0 & 1,0 \\
\hline & 4,8 & 1,9 & 1,8 & 1,6 & 1,3 & 1,2 & 1,1 & 1,0 & 1,0 & 1,0 \\
\hline \multirow[t]{8}{*}{$\geq 5,5<6,5$} & $\geq 6,4$ & 2,1 & 1,9 & 1,7 & 1,4 & 1,2 & 1,1 & 1,0 & 1,0 & 1,0 \\
\hline & 0,4 & 1,2 & 1,1 & 1,1 & 1,0 & 1,0 & 1,0 & 1,0 & 1,0 & 1,0 \\
\hline & 0,8 & 1,5 & 1,4 & 1,3 & 1,2 & 1,1 & 1,0 & 1,0 & 1,0 & 1,0 \\
\hline & 1,2 & 1,6 & 1,5 & 1,4 & 1,2 & 1,1 & 1,1 & 1,0 & 1,0 & 1,0 \\
\hline & 1,6 & 1,7 & 1,6 & 1,5 & 1,2 & 1,1 & 1,1 & 1,0 & 1,0 & 1,0 \\
\hline & 2,4 & 1,7 & 1,6 & 1,6 & 1,3 & 1,2 & 1,1 & 1,0 & 1,0 & 1,0 \\
\hline & 3,2 & 1,8 & 1,7 & 1,6 & 1,3 & 1,2 & 1,1 & 1,0 & 1,0 & 1,0 \\
\hline & 4,8 & 2,1 & 1,9 & 1,7 & 1,4 & 1,2 & 1,1 & 1,0 & 1,0 & 1,0 \\
\hline$\geq 6,5$ & $\geq 6,4$ & 2,4 & 2,1 & 1,8 & 1,4 & 1,2 & 1,1 & 1,0 & 1,0 & 1,0 \\
\hline
\end{tabular}


Tabela 13. Fator de ajuste $f_{n p}$ na determinação de ATS em rampas específicas

\begin{tabular}{|c|c|c|c|c|c|}
\hline \multirow{3}{*}{$\begin{array}{l}\text { Taxa de fluxo } \\
\text { no sentido o- } \\
\text { posto }(c p e / h)\end{array}$} & \multicolumn{5}{|c|}{ Zonas de ultrapassagem proibida (\%) } \\
\hline & $\leq 20$ & 40 & 60 & 80 & 100 \\
\hline & \multicolumn{5}{|c|}{$F F S=110 \mathrm{~km} / \mathrm{h}$} \\
\hline$\leq 100$ & 1,1 & 1,7 & 3,0 & 3,8 & 3,8 \\
\hline 200 & 1,6 & 2,2 & 3,4 & 4,2 & 4,4 \\
\hline 400 & 1,3 & 1,6 & 2,3 & 2,7 & 2,8 \\
\hline 600 & 0,9 & 1,2 & 1,6 & 1,8 & 1,9 \\
\hline 800 & 0,6 & 0,7 & 0,9 & 1,1 & 1,2 \\
\hline 1000 & 0,3 & 0,4 & 0,6 & 0,7 & 0,7 \\
\hline 1200 & 0,2 & 0,2 & 0,3 & 0,4 & 0,4 \\
\hline 1400 & 0,1 & 0,2 & 0,2 & 0,3 & 0,3 \\
\hline \multirow[t]{2}{*}{$\geq 1600$} & 0,0 & 0,0 & 0,1 & 0,1 & 0,1 \\
\hline & \multicolumn{5}{|c|}{$F F S=100 \mathrm{~km} / \mathrm{h}$} \\
\hline$\leq 100$ & 1,2 & 2,0 & 3,1 & 4,8 & 5,0 \\
\hline 200 & 1,7 & 2,6 & 4,1 & 5,4 & 5,8 \\
\hline 400 & 0,9 & 1,6 & 2,3 & 3,2 & 3,5 \\
\hline 600 & 0,6 & 1,0 & 1,6 & 2,1 & 2,3 \\
\hline 800 & 0,4 & 0,7 & 1,1 & 1,4 & 1,5 \\
\hline 1000 & 0,2 & 0,4 & 0,7 & 0,9 & 1,0 \\
\hline 1200 & 0,2 & 0,3 & 0,5 & 0,6 & 0,6 \\
\hline 1400 & 0,1 & 0,1 & 0,2 & 0,3 & 0,3 \\
\hline \multirow[t]{2}{*}{$\geq 1600$} & 0,1 & 0,1 & 0,1 & 0,2 & 0,2 \\
\hline & \multicolumn{5}{|c|}{$F F S=90 \mathrm{~km} / \mathrm{h}$} \\
\hline$\leq 100$ & 0,7 & 1,5 & 2,8 & 5,2 & 5,9 \\
\hline 200 & 1,4 & 2,6 & 4,1 & 5,9 & 6,9 \\
\hline 400 & 1,0 & 1,8 & 2,8 & 3,7 & 4,3 \\
\hline 600 & 0,7 & 1,2 & 1,9 & 2,6 & 2,9 \\
\hline 800 & 0,4 & 0,7 & 1,2 & 1,5 & 1,7 \\
\hline 1000 & 0,3 & 0,6 & 0,9 & 1,1 & 1,3 \\
\hline 1200 & 0,2 & 0,4 & 0,6 & 0,7 & 0,8 \\
\hline 1400 & 0,1 & 0,2 & 0,3 & 0,4 & 0,5 \\
\hline \multirow[t]{2}{*}{$\geq 1600$} & 0,0 & 0,2 & 0,2 & 0,3 & 0,3 \\
\hline & \multicolumn{5}{|c|}{$F F S=80 \mathrm{~km} / \mathrm{h}$} \\
\hline$\leq 100$ & 0,7 & 1,3 & 2,3 & 4,6 & 6,4 \\
\hline 200 & 1,1 & 2,1 & 3,5 & 5,9 & 7,3 \\
\hline 400 & 1,0 & 1,8 & 2,5 & 3,7 & 4,7 \\
\hline 600 & 0,6 & 1,0 & 1,5 & 2,2 & 2,7 \\
\hline 800 & 0,4 & 0,6 & 1,0 & 1,4 & 1,8 \\
\hline 1000 & 0,2 & 0,5 & 0,7 & 1,0 & 1,3 \\
\hline 1200 & 0,2 & 0,3 & 0,5 & 0,7 & 0,8 \\
\hline 1400 & 0,1 & 0,2 & 0,4 & 0,5 & 0,6 \\
\hline \multirow[t]{2}{*}{$\geq 1600$} & 0,1 & 0,1 & 0,2 & 0,3 & 0,3 \\
\hline & \multicolumn{5}{|c|}{$F F S=70 \mathrm{~km} / \mathrm{h}$} \\
\hline$\leq 100$ & 0,5 & 1,2 & 2,3 & 4,7 & 7,6 \\
\hline 200 & 1,1 & 2,0 & 3,9 & 6,1 & 8,2 \\
\hline 400 & 1,0 & 1,9 & 2,9 & 3,9 & 5,2 \\
\hline 600 & 0,5 & 1,0 & 1,7 & 2,3 & 3,0 \\
\hline 800 & 0,2 & 0,5 & 0,9 & 1,4 & 1,8 \\
\hline 1000 & 0,2 & 0,5 & 0,7 & 1,1 & 1,4 \\
\hline 1200 & 0,2 & 0,3 & 0,6 & 0,8 & 0,9 \\
\hline 1400 & 0,1 & 0,2 & 0,4 & 0,5 & 0,6 \\
\hline$\geq 1600$ & 0,1 & 0,2 & 0,2 & 0,3 & 0,3 \\
\hline
\end{tabular}

Tabela 14. Fator de ajuste $f_{n p}$ na determinação de PTSF em rampas específicas

\begin{tabular}{|c|c|c|c|c|c|}
\hline \multirow{3}{*}{$\begin{array}{l}\text { Taxa de fluxo } \\
\text { no sentido o- } \\
\text { posto (cpe/h) }\end{array}$} & \multicolumn{5}{|c|}{ Zonas de ultrapassagem proibida (\%) } \\
\hline & $\leq 20$ & 40 & 60 & 80 & 100 \\
\hline & \multicolumn{5}{|c|}{$F F S=110 \mathrm{~km} / \mathrm{h}$} \\
\hline$\leq 100$ & 5,0 & 7,7 & 12,5 & 15,7 & 15,9 \\
\hline 200 & 6,4 & 9,5 & 14,5 & 17,6 & 18,2 \\
\hline 400 & 4,9 & 6,8 & 9,3 & 10,8 & 11,2 \\
\hline 600 & 3,1 & 4,0 & 5,7 & 6,6 & 6,9 \\
\hline 800 & 1,7 & 2,3 & 3,0 & 3,6 & 3,9 \\
\hline 1000 & 0,9 & 1,3 & 1,7 & 2,0 & 2,1 \\
\hline 1200 & 0,4 & 0,6 & 0,8 & 0,9 & 1,0 \\
\hline 1400 & 0,1 & 0,2 & 0,3 & 0,4 & 0,4 \\
\hline \multirow[t]{2}{*}{$\geq 1600$} & 0,1 & 0,1 & 0,1 & 0,2 & 0,2 \\
\hline & \multicolumn{5}{|c|}{$F F S=100 \mathrm{~km} / \mathrm{h}$} \\
\hline$\leq 100$ & 6,0 & 9,3 & 14,4 & 20,8 & 22,5 \\
\hline 200 & 8,1 & 11,9 & 18,3 & 23,5 & 25,4 \\
\hline 400 & 3,4 & 6,1 & 9,6 & 13,0 & 14,1 \\
\hline 600 & 2,1 & 3,6 & 6,2 & 7,8 & 8,6 \\
\hline 800 & 1,5 & 2,4 & 3,6 & 4,5 & 5,1 \\
\hline 1000 & 0,8 & 1,3 & 2,0 & 2,6 & 2,8 \\
\hline 1200 & 0,3 & 0,7 & 1,0 & 1,3 & 1,5 \\
\hline 1400 & 0,2 & 0,3 & 0,4 & 0,5 & 0,6 \\
\hline \multirow[t]{2}{*}{$\geq 1600$} & 0,1 & 0,1 & 0,2 & 0,3 & 0,3 \\
\hline & \multicolumn{5}{|c|}{$F F S=90 \mathrm{~km} / \mathrm{h}$} \\
\hline$\leq 100$ & 3,5 & 7,4 & 13,7 & 23,5 & 26,9 \\
\hline 200 & 6,4 & 11,6 & 18,5 & 26,4 & 30,2 \\
\hline 400 & 4,3 & 7,2 & 10,0 & 15,0 & 17,4 \\
\hline 600 & 2,6 & 4,4 & 6,8 & 9,3 & 10,5 \\
\hline 800 & 1,5 & 1,5 & 2,6 & 4,0 & 4,9 \\
\hline 1000 & 1,0 & 1,8 & 2,5 & 3,3 & 3,7 \\
\hline 1200 & 0,6 & 1,0 & 1,4 & 1,7 & 2,0 \\
\hline 1400 & 0,2 & 0,5 & 0,7 & 1,0 & 1,1 \\
\hline \multirow[t]{2}{*}{$\geq 1600$} & 0,2 & 0,3 & 0,3 & 0,4 & 0,5 \\
\hline & \multicolumn{5}{|c|}{$F F S=80 \mathrm{~km} / \mathrm{h}$} \\
\hline$\leq 100$ & 4,0 & 7,1 & 12,6 & 23,9 & 31,8 \\
\hline$\overline{2} 00$ & 6,5 & 10,8 & 17,4 & 29,1 & 35,0 \\
\hline 400 & 4,7 & 7,4 & 10,6 & 15,8 & 19,6 \\
\hline 600 & 2,0 & 3,8 & 5,9 & 8,5 & 10,6 \\
\hline 800 & 1,4 & 2,0 & 3,4 & 4,8 & 6,2 \\
\hline 1000 & 0,8 & 1,6 & 2,2 & 3,2 & 3,9 \\
\hline 1200 & 0,5 & 1,1 & 1,5 & 1,9 & 2,3 \\
\hline 1400 & 0,3 & 0,5 & 0,8 & 1,1 & 1,3 \\
\hline \multirow[t]{2}{*}{$\geq 1600$} & 0,2 & 0,3 & 0,4 & 0,5 & 0,6 \\
\hline & \multicolumn{5}{|c|}{$F F S=70 \mathrm{~km} / \mathrm{h}$} \\
\hline$\leq 100$ & 3,1 & 6,7 & 12,3 & 23,9 & 37,8 \\
\hline 200 & 5,2 & 9,2 & 18,3 & 28,5 & 38,6 \\
\hline 400 & 4,4 & 7,9 & 12,0 & 16,4 & 21,6 \\
\hline 600 & 1,6 & 4,0 & 5,9 & 8,5 & 11,0 \\
\hline 800 & 0,5 & 1,6 & 2,9 & 4,5 & 5,9 \\
\hline 1000 & 0,4 & 1,4 & 2,2 & 3,1 & 3,9 \\
\hline 1200 & 0,3 & 0,9 & 1,4 & 1,8 & 2,2 \\
\hline 1400 & 0,3 & 0,5 & 0,7 & 0,9 & 1,1 \\
\hline$\geq 1600$ & 0,1 & 0,2 & 0,3 & 0,4 & 0,4 \\
\hline
\end{tabular}


Tabela 15. Fator de ajuste para o efeito das faixas de ultrapassagem $\left(f_{p l}\right)$ na determinação de $A T S_{p l}$ e $P T S F_{p l}$

\begin{tabular}{|c|c|c|}
\hline $\begin{array}{l}\text { Taxa de fluxo em } \\
\text { sentido único (cpe/h) }\end{array}$ & $\begin{array}{l}\text { Estimação de } \\
\text { ATS }_{P L}\end{array}$ & $\begin{array}{l}\text { Estimação de } \\
\text { PTSF }_{p l}\end{array}$ \\
\hline $0-300$ & 1,07 & 0,31 \\
\hline $300-600$ & 1,10 & 0,40 \\
\hline$>600$ & 1,14 & 0,45 \\
\hline
\end{tabular}

Tabela 16. Fator de ajuste para o efeito das faixas adicionais em subidas $\left(f_{p l}\right)$ na determinação de $A T S_{P L}$

\begin{tabular}{|c|c|c|c|c|c|c|c|c|c|c|c|c|c|}
\hline \multirow{4}{*}{$\begin{array}{l}\text { Declividade } \\
\text { (\%) }\end{array}$} & \multirow{4}{*}{$\begin{array}{l}L \\
(\mathrm{~km})\end{array}$} & \multicolumn{4}{|c|}{$\begin{array}{l}\text { Taxa de fluxo em } \\
\text { sentido único (cpe/h) }\end{array}$} & \multicolumn{4}{|c|}{$\begin{array}{l}\text { Taxa de fluxo em } \\
\text { sentido único (cpe/h) }\end{array}$} & \multicolumn{4}{|c|}{$\begin{array}{l}\text { Taxa de fluxo em } \\
\text { sentido único }(\mathrm{cpe} / \mathrm{h})\end{array}$} \\
\hline & & \multicolumn{4}{|c|}{$0-300$} & \multicolumn{4}{|c|}{$>300-600$} & \multicolumn{4}{|c|}{$>600$} \\
\hline & & \multicolumn{4}{|c|}{ Caminhões (\%) } & \multicolumn{4}{|c|}{ Caminhões (\%) } & \multicolumn{4}{|c|}{ Caminhões (\%) } \\
\hline & & 0 & 20 & 30 & 40 & 0 & 20 & 30 & 40 & 0 & 20 & 30 & 40 \\
\hline \multirow[t]{8}{*}{$\geq 3,0<3,5$} & 0,4 & 1,00 & 1,00 & 1,00 & 1,00 & 1,00 & 1,02 & 1,02 & 1,02 & 1,01 & 1,01 & 1,01 & 1,03 \\
\hline & 0,8 & 1,00 & 1,00 & 1,00 & 1,00 & 1,00 & 1,02 & 1,02 & 1,03 & 1,02 & 1,07 & 1,07 & 1,07 \\
\hline & 1,2 & 1,00 & 1,00 & 1,00 & 1,00 & 1,00 & 1,03 & 1,03 & 1,04 & 1,03 & 1,09 & 1,09 & 1,09 \\
\hline & 1,6 & 1,00 & 1,00 & 1,00 & 1,00 & 1,00 & 1,03 & 1,03 & 1,04 & 1,03 & 1,10 & 1,10 & 1,10 \\
\hline & 2,4 & 1,00 & 1,00 & 1,01 & 1,01 & 1,00 & 1,03 & 1,03 & 1,04 & 1,04 & 1,14 & 1,14 & 1,14 \\
\hline & 3,2 & 1,00 & 1,00 & 1,01 & 1,01 & 1,00 & 1,03 & 1,04 & 1,04 & 1,05 & 1,15 & 1,15 & 1,15 \\
\hline & 4,8 & 1,00 & 1,00 & 1,01 & 1,01 & 1,02 & 1,03 & 1,04 & 1,05 & 1,06 & 1,15 & 1,15 & 1,18 \\
\hline & $\geq 6,4$ & 1,00 & 1,00 & 1,01 & 1,01 & 1,02 & 1,03 & 1,04 & 1,05 & 1,07 & 1,17 & 1,19 & 1,19 \\
\hline \multirow[t]{8}{*}{$\geq 3,5<4,5$} & 0,4 & 1,00 & 1,00 & 1,00 & 1,00 & 1,00 & 1,02 & 1,02 & 1,02 & 1,02 & 1,04 & 1,04 & 1,04 \\
\hline & 0,8 & 1,00 & 1,00 & 1,00 & 1,01 & 1,00 & 1,02 & 1,02 & 1,04 & 1,03 & 1,09 & 1,09 & 1,09 \\
\hline & 1,2 & 1,00 & 1,00 & 1,00 & 1,01 & 1,00 & 1,03 & 1,03 & 1,04 & 1,03 & 1,10 & 1,10 & 1,12 \\
\hline & 1,6 & 1,00 & 1,00 & 1,01 & 1,01 & 1,00 & 1,03 & 1,04 & 1,04 & 1,05 & 1,13 & 1,13 & 1,14 \\
\hline & 2,4 & 1,00 & 1,01 & 1,01 & 1,01 & 1,00 & 1,03 & 1,04 & 1,05 & 1,05 & 1,16 & 1,16 & 1,16 \\
\hline & 3,2 & 1,00 & 1,01 & 1,01 & 1,01 & 1,00 & 1,03 & 1,04 & 1,05 & 1,07 & 1,17 & 1,17 & 1,17 \\
\hline & 4,8 & 1,00 & 1,01 & 1,01 & 1,01 & 1,02 & 1,03 & 1,05 & 1,05 & 1,07 & 1,17 & 1,18 & 1,18 \\
\hline & $\geq 6,4$ & 1,00 & 1,01 & 1,01 & 1,01 & 1,02 & 1,03 & 1,05 & 1,05 & 1,07 & 1,18 & 1,19 & 1,19 \\
\hline \multirow[t]{8}{*}{$\geq 4,5<5,5$} & 0,4 & 1,00 & 1,00 & 1,00 & 1,01 & 1,00 & 1,02 & 1,02 & 1,03 & 1,03 & 1,06 & 1,06 & 1,06 \\
\hline & 0,8 & 1,00 & 1,00 & 1,00 & 1,01 & 1,00 & 1,02 & 1,03 & 1,03 & 1,03 & 1,09 & 1,09 & 1,10 \\
\hline & 1,2 & 1,00 & 1,00 & 1,01 & 1,02 & 1,00 & 1,03 & 1,03 & 1,04 & 1,03 & 1,10 & 1,10 & 1,12 \\
\hline & 1,6 & 1,00 & 1,00 & 1,01 & 1,02 & 1,00 & 1,03 & 1,03 & 1,04 & 1,05 & 1,13 & 1,15 & 1,15 \\
\hline & 2,4 & 1,00 & 1,01 & 1,02 & 1,02 & 1,00 & 1,03 & 1,04 & 1,05 & 1,06 & 1,16 & 1,16 & 1,17 \\
\hline & 3,2 & 1,00 & 1,01 & 1,02 & 1,02 & 1,02 & 1,04 & 1,04 & 1,05 & 1,07 & 1,17 & 1,17 & 1,19 \\
\hline & 4,8 & 1,00 & 1,01 & 1,02 & 1,02 & 1,02 & 1,04 & 1,05 & 1,05 & 1,07 & 1,18 & 1,18 & 1,20 \\
\hline & $\geq 6,4$ & 1,00 & 1,01 & 1,02 & 1,02 & 1,02 & 1,04 & 1,05 & 1,05 & 1,07 & 1,18 & 1,19 & 1,20 \\
\hline \multirow[t]{8}{*}{$\geq 5,5<6,5$} & 0,4 & 1,00 & 1,00 & 1,00 & 1,01 & 1,00 & 1,03 & 1,03 & 1,04 & 1,04 & 1,07 & 1,07 & 1,07 \\
\hline & 0,8 & 1,00 & 1,00 & 1,00 & 1,02 & 1,00 & 1,03 & 1,03 & 1,04 & 1,04 & 1,10 & 1,10 & 1,11 \\
\hline & 1,2 & 1,00 & 1,00 & 1,01 & 1,02 & 1,00 & 1,03 & 1,04 & 1,04 & 1,05 & 1,12 & 1,13 & 1,13 \\
\hline & 1,6 & 1,00 & 1,00 & 1,01 & 1,02 & 1,00 & 1,03 & 1,04 & 1,05 & 1,06 & 1,14 & 1,15 & 1,15 \\
\hline & 2,4 & 1,00 & 1,01 & 1,02 & 1,02 & 1,02 & 1,04 & 1,05 & 1,05 & 1,06 & 1,16 & 1,16 & 1,17 \\
\hline & 3,2 & 1,00 & 1,01 & 1,02 & 1,02 & 1,02 & 1,04 & 1,05 & 1,05 & 1,07 & 1,17 & 1,17 & 1,19 \\
\hline & 4,8 & 1,00 & 1,01 & 1,02 & 1,02 & 1,02 & 1,04 & 1,06 & 1,06 & 1,07 & 1,18 & 1,18 & 1,20 \\
\hline & $\geq 6,4$ & 1,00 & 1,01 & 1,02 & 1,02 & 1,03 & 1,04 & 1,06 & 1,06 & 1,07 & 1,19 & 1,19 & 1,20 \\
\hline \multirow[t]{8}{*}{$\geq 6,5$} & 0,4 & 1,00 & 1,00 & 1,01 & 1,01 & 1,00 & 1,04 & 1,04 & 1,05 & 1,04 & 1,07 & 1,08 & 1,08 \\
\hline & 0,8 & 1,00 & 1,01 & 1,01 & 1,02 & 1,00 & 1,04 & 1,04 & 1,05 & 1,05 & 1,10 & 1,10 & 1,12 \\
\hline & 1,2 & 1,00 & 1,01 & 1,01 & 1,02 & 1,00 & 1,04 & 1,04 & 1,05 & 1,05 & 1,12 & 1,13 & 1,13 \\
\hline & 1,6 & 1,00 & 1,01 & 1,02 & 1,02 & 1,02 & 1,04 & 1,05 & 1,05 & 1,06 & 1,13 & 1,15 & 1,15 \\
\hline & 2,4 & 1,00 & 1,01 & 1,02 & 1,02 & 1,02 & 1,05 & 1,05 & 1,05 & 1,06 & 1,14 & 1,16 & 1,17 \\
\hline & 3,2 & 1,00 & 1,01 & 1,02 & 1,02 & 1,02 & 1,05 & 1,05 & 1,06 & 1,07 & 1,16 & 1,17 & 1,19 \\
\hline & 4,8 & 1,00 & 1,01 & 1,02 & 1,02 & 1,03 & 1,05 & 1,06 & 1,06 & 1,07 & 1,17 & 1,18 & 1,20 \\
\hline & $\geq 6,4$ & 1,00 & 1,01 & 1,02 & 1,02 & 1,03 & 1,05 & 1,06 & 1,06 & 1,07 & 1,18 & 1,19 & 1,21 \\
\hline
\end{tabular}


Tabela 17. Fator de ajuste para o efeito das faixas adicionais em subidas $\left(f_{p l}\right)$ na determinação de $P T S F_{P L}$

\begin{tabular}{|c|c|c|c|c|c|c|c|c|c|c|c|c|c|}
\hline \multirow{4}{*}{$\begin{array}{l}\text { Declividade } \\
\text { (\%) }\end{array}$} & \multirow{4}{*}{$\begin{array}{l}L \\
(\mathrm{~km})\end{array}$} & \multicolumn{4}{|c|}{$\begin{array}{l}\text { Taxa de fluxo em } \\
\text { sentido único (cpe/h) }\end{array}$} & \multicolumn{4}{|c|}{$\begin{array}{l}\text { Taxa de fluxo em } \\
\text { sentido único (cpe/h) }\end{array}$} & \multicolumn{4}{|c|}{$\begin{array}{l}\text { Taxa de fluxo em } \\
\text { sentido único }(\text { cpe/h) }\end{array}$} \\
\hline & & \multicolumn{4}{|c|}{$0-300$} & \multicolumn{4}{|c|}{$>300-600$} & \multicolumn{4}{|c|}{$>600$} \\
\hline & & \multicolumn{4}{|c|}{ Caminhões (\%) } & \multicolumn{4}{|c|}{ Caminhões (\%) } & \multicolumn{4}{|c|}{ Caminhões (\%) } \\
\hline & & 0 & 20 & 30 & 40 & 0 & 20 & 30 & 40 & 0 & 20 & 30 & 40 \\
\hline & 0,4 & 0,82 & 0,77 & 0,72 & 0,67 & 0,83 & 0,74 & 0,73 & 0,71 & 0,83 & 0,76 & 0,75 & 0,75 \\
\hline & 0,8 & 0,60 & 0,55 & 0,53 & 0,52 & 0,62 & 0,55 & 0,54 & 0,53 & 0,62 & 0,61 & 0,67 & 0,67 \\
\hline & 1,2 & 0,51 & 0,41 & 0,41 & 0,41 & 0,52 & 0,44 & 0,45 & 0,46 & 0,52 & 0,53 & 0,60 & 0,62 \\
\hline & 1,6 & 0,44 & 0,33 & 0,35 & 0,37 & 0,48 & 0,40 & 0,40 & 0,40 & 0,48 & 0,51 & 0,58 & 0,58 \\
\hline & 2,4 & 0,34 & 0,30 & 0,30 & 0,30 & 0,41 & 0,34 & 0,34 & 0,34 & 0,43 & 0,45 & 0,58 & 0,56 \\
\hline & 3,2 & 0,30 & 0,23 & 0,26 & 0,27 & 0,35 & 0,29 & 0,30 & 0,32 & 0,39 & 0,44 & 0,56 & 0,56 \\
\hline & 4,8 & 0,28 & 0,20 & 0,22 & 0,27 & 0,31 & 0,25 & 0,25 & 0,28 & 0,36 & 0,41 & 0,56 & 0,56 \\
\hline \multirow[t]{8}{*}{$\geq 3,0<3,5$} & $\geq 6,4$ & 0,25 & 0,18 & 0,21 & 0,27 & 0,28 & 0,24 & 0,25 & 0,26 & 0,34 & 0,40 & 0,56 & 0,56 \\
\hline & 0,4 & 0,77 & 0,68 & 0,66 & 0,64 & 0,77 & 0,69 & 0,67 & 0,66 & 0,77 & 0,74 & 0,68 & 0,68 \\
\hline & 0,8 & 0,60 & 0,42 & 0,50 & 0,52 & 0,62 & 0,52 & 0,52 & 0,50 & 0,62 & 0,61 & 0,61 & 0,61 \\
\hline & 1,2 & 0,46 & 0,39 & 0,41 & 0,41 & 0,50 & 0,43 & 0,42 & 0,40 & 0,50 & 0,53 & 0,60 & 0,60 \\
\hline & 1,6 & 0,44 & 0,31 & 0,32 & 0,33 & 0,44 & 0,38 & 0,38 & 0,40 & 0,45 & 0,51 & 0,58 & 0,56 \\
\hline & 2,4 & 0,34 & 0,29 & 0,30 & 0,30 & 0,36 & 0,32 & 0,34 & 0,34 & 0,40 & 0,45 & 0,56 & 0,56 \\
\hline & 3,2 & 0,29 & 0,23 & 0,26 & 0,27 & 0,33 & 0,29 & 0,30 & 0,32 & 0,38 & 0,49 & 0,56 & 0,56 \\
\hline & 4,8 & 0,24 & 0,20 & 0,22 & 0,27 & 0,28 & 0,25 & 0,25 & 0,28 & 0,33 & 0,41 & 0,56 & 0,56 \\
\hline \multirow[t]{8}{*}{$\geq 3,5<4,5$} & $\geq 6,4$ & 0,24 & 0,18 & 0,21 & 0,24 & 0,24 & 0,24 & 0,25 & 0,26 & 0,33 & 0,40 & 0,56 & 0,56 \\
\hline & 0,4 & 0,72 & 0,66 & 0,60 & 0,59 & 0,75 & 0,60 & 0,63 & 0,62 & 0,77 & 0,71 & 0,64 & 0,64 \\
\hline & 0,8 & 0,56 & 0,44 & 0,49 & 0,49 & 0,56 & 0,48 & 0,51 & 0,50 & 0,56 & 0,58 & 0,61 & 0,61 \\
\hline & 1,2 & 0,46 & 0,37 & 0,40 & 0,40 & 0,47 & 0,40 & 0,42 & 0,40 & 0,47 & 0,53 & 0,60 & 0,60 \\
\hline & 1,6 & 0,36 & 0,31 & 0,32 & 0,33 & 0,36 & 0,37 & 0,38 & 0,40 & 0,43 & 0,51 & 0,56 & 0,56 \\
\hline & 2,4 & 0,33 & 0,29 & 0,30 & 0,30 & 0,34 & 0,32 & 0,34 & 0,34 & 0,39 & 0,45 & 0,56 & 0,56 \\
\hline & 3,2 & 0,23 & 0,23 & 0,26 & 0,26 & 0,30 & 0,29 & 0,30 & 0,32 & 0,36 & 0,44 & 0,56 & 0,56 \\
\hline & 4,8 & 0,22 & 0,20 & 0,22 & 0,24 & 0,27 & 0,29 & 0,25 & 0,28 & 0,33 & 0,41 & 0,56 & 0,56 \\
\hline \multirow[t]{8}{*}{$\geq 4,5<5,5$} & $\geq 6,4$ & 0,22 & 0,18 & 0,21 & 0,24 & 0,23 & 0,24 & 0,25 & 0,26 & 0,32 & 0,40 & 0,56 & 0,56 \\
\hline & 0,4 & 0,63 & 0,60 & 0,57 & 0,54 & 0,63 & 0,60 & 0,59 & 0,59 & 0,63 & 0,66 & 0,62 & 0,62 \\
\hline & 0,8 & 0,52 & 0,43 & 0,43 & 0,39 & 0,52 & 0,43 & 0,51 & 0,50 & 0,52 & 0,58 & 0,60 & 0,60 \\
\hline & 1,2 & 0,41 & 0,32 & 0,33 & 0,33 & 0,41 & 0,37 & 0,42 & 0,40 & 0,44 & 0,53 & 0,60 & 0,60 \\
\hline & 1,6 & 0,36 & 0,31 & 0,31 & 0,31 & 0,36 & 0,36 & 0,37 & 0,40 & 0,41 & 0,51 & 0,56 & 0,56 \\
\hline & 2,4 & 0,31 & 0,29 & 0,29 & 0,29 & 0,31 & 0,32 & 0,34 & 0,34 & 0,37 & 0,45 & 0,56 & 0,56 \\
\hline & 3,2 & 0,23 & 0,23 & 0,26 & 0,26 & 0,25 & 0,29 & 0,30 & 0,32 & 0,36 & 0,44 & 0,56 & 0,56 \\
\hline & 4,8 & 0,22 & 0,20 & 0,22 & 0,24 & 0,24 & 0,25 & 0,25 & 0,28 & 0,33 & 0,41 & 0,56 & 0,56 \\
\hline \multirow[t]{8}{*}{$\geq 5,5<6,5$} & $\geq 6,4$ & 0,21 & 0,18 & 0,21 & 0,24 & 0,21 & 0,24 & 0,25 & 0,26 & 0,32 & 0,40 & 0,56 & 0,56 \\
\hline & 0,4 & 0,58 & 0,52 & 0,49 & 0,49 & 0,58 & 0,56 & 0,56 & 0,56 & 0,60 & 0,67 & 0,60 & 0,60 \\
\hline & 0,8 & 0,46 & 0,31 & 0,36 & 0,39 & 0,46 & 0,41 & 0,44 & 0,50 & 0,49 & 0,58 & 0,60 & 0,60 \\
\hline & 1,2 & 0,38 & 0,31 & 0,33 & 0,33 & 0,38 & 0,37 & 0,38 & 0,40 & 0,43 & 0,53 & 0,60 & 0,60 \\
\hline & 1,6 & 0,34 & 0,31 & 0,31 & 0,31 & 0,34 & 0,36 & 0,37 & 0,39 & 0,40 & 0,51 & 0,56 & 0,56 \\
\hline & 2,4 & 0,28 & 0,26 & 0,39 & 0,29 & 0,28 & 0,32 & 0,34 & 0,34 & 0,36 & 0,45 & 0,56 & 0,56 \\
\hline & 3,2 & 0,23 & 0,23 & 0,26 & 0,26 & 0,25 & 0,29 & 0,30 & 0,32 & 0,34 & 0,44 & 0,56 & 0,56 \\
\hline & 4,8 & 0,22 & 0,20 & 0,22 & 0,24 & 0,22 & 0,25 & 0,25 & 0,28 & 0,33 & 0,41 & 0,56 & 0,56 \\
\hline$\geq 6,5$ & $\geq 6,4$ & 0,20 & 0,18 & 0,21 & 0,24 & 0,20 & 0,24 & 0,25 & 0,26 & 0,31 & 0,40 & 0,56 & 0,56 \\
\hline
\end{tabular}


Tabela 18. Comparação entre os níveis de serviço observados e estimados

\begin{tabular}{|c|c|c|c|c|c|c|c|}
\hline \multirow[b]{2}{*}{ Amostra } & \multicolumn{3}{|c|}{ Nível de serviço } & \multirow[b]{2}{*}{ Amostra } & \multicolumn{3}{|c|}{ Nível de serviço } \\
\hline & Observado & HCM2000 & $\begin{array}{l}\text { HCM } \\
\text { adaptado }\end{array}$ & & Observado & HCM2000 & $\begin{array}{l}\text { HCM } \\
\text { adaptado }\end{array}$ \\
\hline \multicolumn{4}{|c|}{ SP-215, km 88 a km 93} & \multicolumn{4}{|c|}{ SP-215, km 107 a km 111} \\
\hline 1 & $\mathrm{~B}$ & $\mathrm{C}$ & $\mathrm{B}$ & 1 & $\mathrm{~B}$ & $\mathrm{C}$ & $\mathrm{B}$ \\
\hline 2 & $\mathrm{~B}$ & $\mathrm{C}$ & B & 2 & $\mathrm{~B}$ & $\mathrm{C}$ & $\mathrm{B}$ \\
\hline 3 & $\mathrm{~B}$ & $\mathrm{~B}$ & $\mathrm{~B}$ & 3 & $\mathrm{~B}$ & $\mathrm{C}$ & $\mathrm{B}$ \\
\hline 4 & $\mathrm{~B}$ & $\mathrm{~B}$ & $\mathrm{~B}$ & 4 & $\mathrm{C}$ & $\mathrm{C}$ & $\mathrm{B}$ \\
\hline 5 & $\mathrm{~B}$ & $\mathrm{C}$ & $\mathrm{B}$ & 5 & $\mathrm{C}$ & $\mathrm{C}$ & $\mathrm{B}$ \\
\hline 6 & $\mathrm{~B}$ & $\mathrm{C}$ & $\mathrm{B}$ & 6 & $\mathrm{C}$ & $\mathrm{C}$ & $\mathrm{B}$ \\
\hline \multicolumn{4}{|c|}{ SP-215, km 133 a km 138,5 } & \multicolumn{4}{|c|}{$\mathrm{SP}-225, \mathrm{~km} 113$ a km 121} \\
\hline 1 & $\mathrm{~B}$ & $\mathrm{C}$ & $\mathrm{B}$ & 1 & $\mathrm{~B}$ & $\mathrm{C}$ & $\mathrm{B}$ \\
\hline 2 & $\mathrm{~B}$ & $\mathrm{C}$ & $\mathrm{B}$ & 2 & $\mathrm{~B}$ & $\mathrm{C}$ & $\mathrm{B}$ \\
\hline 3 & $\mathrm{~B}$ & $\mathrm{C}$ & $\mathrm{B}$ & 3 & $\mathrm{~B}$ & $\mathrm{C}$ & $\mathrm{B}$ \\
\hline 4 & $\mathrm{~B}$ & $\mathrm{C}$ & B & 4 & $\mathrm{~B}$ & $\mathrm{C}$ & $\mathrm{B}$ \\
\hline 5 & $\mathrm{~B}$ & $\mathrm{C}$ & $\mathrm{B}$ & 5 & $\mathrm{~B}$ & $\mathrm{C}$ & $\mathrm{B}$ \\
\hline 6 & $\mathrm{~B}$ & $\mathrm{C}$ & $\mathrm{B}$ & 6 & $\mathrm{C}$ & $\mathrm{C}$ & $\mathrm{B}$ \\
\hline 7 & $\mathrm{~B}$ & $\mathrm{C}$ & $\mathrm{B}$ & 7 & $\mathrm{C}$ & $\mathrm{C}$ & $\mathrm{B}$ \\
\hline 8 & $\mathrm{~B}$ & $\mathrm{C}$ & $\mathrm{B}$ & 8 & $\mathrm{~B}$ & $\mathrm{C}$ & $\mathrm{B}$ \\
\hline \multicolumn{4}{|c|}{ SP-225, km 133 a km 141} & \multicolumn{4}{|c|}{ SP-253, km 151 a km 155} \\
\hline 1 & $\mathrm{~B}$ & $\mathrm{~B}$ & $\mathrm{~B}$ & 1 & $\mathrm{~B}$ & $\mathrm{~A}$ & $\mathrm{~B}$ \\
\hline 2 & $\mathrm{~B}$ & $\mathrm{~B}$ & $\mathrm{~B}$ & 2 & $\mathrm{~B}$ & A & $\mathrm{B}$ \\
\hline 3 & $\mathrm{~B}$ & $\mathrm{~B}$ & $\mathrm{~B}$ & 3 & $\mathrm{~B}$ & $\mathrm{~A}$ & $\mathrm{~B}$ \\
\hline 4 & $\mathrm{~B}$ & $\mathrm{~B}$ & $\mathrm{~B}$ & 4 & $\mathrm{~B}$ & A & $\mathrm{B}$ \\
\hline 5 & $\mathrm{~B}$ & $\mathrm{C}$ & $\mathrm{B}$ & 5 & $\mathrm{~B}$ & A & $\mathrm{B}$ \\
\hline 6 & $\mathrm{~B}$ & $\mathrm{C}$ & $\mathrm{B}$ & 6 & $\mathrm{~B}$ & $\mathrm{~A}$ & $\mathrm{~B}$ \\
\hline 7 & $\mathrm{~B}$ & $\mathrm{C}$ & $\mathrm{B}$ & 7 & $\mathrm{~B}$ & $\mathrm{~A}$ & $\mathrm{C}$ \\
\hline 8 & $\mathrm{C}$ & $\mathrm{C}$ & $\mathrm{B}$ & 8 & $\mathrm{C}$ & $\mathrm{B}$ & $\mathrm{C}$ \\
\hline \multicolumn{8}{|c|}{ SP-322, km 382 a km 388} \\
\hline 1 & $\mathrm{~B}$ & $\mathrm{~B}$ & B & & & & \\
\hline 2 & $\mathrm{~B}$ & $\mathrm{C}$ & B & & & & \\
\hline 3 & B & $\mathrm{C}$ & B & & & & \\
\hline 4 & B & $\mathrm{C}$ & B & & & & \\
\hline 5 & B & $\mathrm{C}$ & B & & & & \\
\hline 6 & $\mathrm{~B}$ & $\mathrm{C}$ & B & & & & \\
\hline 7 & $\mathrm{~B}$ & $\mathrm{C}$ & B & & & & \\
\hline 8 & $\mathrm{~B}$ & $\mathrm{C}$ & $\mathrm{B}$ & & & & \\
\hline
\end{tabular}

